DOI 10.4171/JEMS/323

Karl Auinger $\cdot$ Igor Dolinka $\cdot$ Mikhail V. Volkov

\title{
Matrix identities involving multiplication and transposition
}

Received April 28, 2009 and in revised form February 18, 2011

\begin{abstract}
We study matrix identities involving multiplication and unary operations such as transposition or Moore-Penrose inversion. We prove that in many cases such identities admit no finite basis.
\end{abstract}

Keywords. Matrix transposition, symplectic transpose, Moore-Penrose inverse, matrix law, identity basis, finite basis problem

\section{Background and motivation}

Matrices $^{1}$ and matrix operations constitute basic tools for many branches of mathematics. Important properties of matrix operations are often expressed in the form of laws or identities such as the associative law for multiplication of matrices. Studying matrix identities that involve multiplication and addition is a classic research direction that was motivated by several important problems in geometry and algebra (see [1] for a survey of the origins of the theory) and that has eventually led to the profound and beautiful theory of PI-rings [14, 21, 23, 46]. Matrix identities involving along with multiplication and addition also certain involution operations (such as taking the usual or symplectic transpose of a matrix) have attracted much attention as well: see, for instance, [9, 10, 15, 20, 46].

If one aims to classify matrix identities of a certain type, then a natural approach is to look for a collection of 'basic' identities such that all other identities would follow from these basic identities. Such a collection is usually referred to as an identity basis or simply a basis. For instance, all identities for matrices over an infinite field involving only multiplication are known to follow from the associative law (see [22, Lemma 2]). Thus, the associative law forms a basis of such 'multiplicative' identities. For identities

K. Auinger: Fakultät für Mathematik, Universität Wien, Nordbergstrasse 15, A-1090 Wien, Austria; e-mail: karl.auinger@univie.ac.at

I. Dolinka: Department of Mathematics and Informatics, University of Novi Sad, Trg Dositeja Obradovića 4, 21101 Novi Sad, Serbia; e-mail: dockie@dmi.uns.ac.rs

M. V. Volkov: Institute of Mathematics and Computer Science, Ural Federal University, Lenina 51, 620000 Ekaterinburg, Russia; e-mail: mikhail.volkov@ usu.ru

Mathematics Subject Classification (2010): Primary 20M07; Secondary 03C05, 15A24, 20M25

1 In this paper, the word 'matrix' always means a square matrix of finite size; also, in order to avoid trivialities, we always assume that the size is at least two. 
involving both multiplication and addition, an explicit basis is known for $2 \times 2$-matrices (except the case of an infinite ground field of characteristic 2) (see [8, 13, 29, 35, 45]), and for $3 \times 3$ - and $4 \times 4$-matrices over a finite field (see [17, 18]). However, for matrices of arbitrary size over a finite field or a field of characteristic 0 the powerful results by Kruse-L'vov [30, 33] and Kemer [24, 25] ensure at least the existence of a finite identity basis for such identities.

In contrast, multiplicative identities for matrices over a finite field admit no finite basis. This rather surprising fact was proved in the mid-1980s by the third author [53, Proposition 3] and Sapir [48, Corollary 6.2]. It is worth noting that the methods used in [53] and [48] were very different but each of them sufficed to cover multiplicative identities for matrices of every fixed size over every finite field.

In the present paper we study matrix identities involving multiplication and one or two natural one-place operations such as taking various transposes or Moore-Penrose inversion. For this we first have to adapt the methods of [53] and [48]. We present the corresponding results in Section 2 while Section 1 collects necessary preliminaries. Applications to the finite basis problem for matrix identities are presented in Section 3. Both methods of Section 2 are used here, and it turns out that they in some sense complement one another since, in contrast to the case of purely multiplicative identities, none of the methods alone suffices to cover, say, identities for matrices of every size over every finite field involving multiplication and the usual transposition of matrices.

Our main results may be summarized as follows.

Theorem. None of the following sets of matrix identities admit a finite identity basis:

- the identities for $n \times n$-matrices over a finite field involving multiplication and usual transposition;

- the identities for $2 n \times 2 n$-matrices over a finite field involving multiplication and symplectic transposition;

- the identities for $2 \times 2$-matrices over the field of complex numbers involving either multiplication and Moore-Penrose inversion or multiplication, Moore-Penrose inversion and Hermitian conjugation;

- the identities for Boolean $n \times n$-matrices involving multiplication and transposition.

We mention in passing that the tools developed in Section 2 admit many further applications that will be published in a separate paper.

As far as the theory of matrices is concerned, we use fairly standard concepts of linear algebra (see [37]). Our proofs involve however some notions of equational logic and semigroup theory which are briefly recalled in the next section.

\section{Preliminaries}

\subsection{Vocabulary of equational logic}

The concepts of an identity and an identity basis are intuitively clear. Nevertheless, any precise reasoning about these concepts requires a formal framework, especially when one aims at 'negative' results as we do in this paper. Such a framework, provided by equational 
logic, is concisely presented, e.g., in [5, Chapter II]. For the reader's convenience, we briefly overview the basic vocabulary of equational logic in a form adapted to the use in this paper. Readers familiar with equational logic may, of course, skip this overview.

A non-empty set $A$ endowed with operations

$$
f_{1}: \underbrace{A \times \cdots \times A}_{n_{1}} \rightarrow A, \quad f_{2}: \underbrace{A \times \cdots \times A}_{n_{2}} \rightarrow A, \quad \cdots
$$

is called an algebraic structure of type $\left(n_{1}, n_{2}, \ldots\right)$ with the carrier A. Algebraic structures considered in this paper are mostly of types $(2,1)$ or $(2,1,1)$ so that they have one binary operation and one or two unary operations. Since the binary operations involved are always assumed to satisfy the associative law, our structures are semigroups equipped with one or two additional unary operations; for brevity, we shall call such structures unary semigroups.

We formally define notions related to unary semigroup identities only for the case of one unary operation, leaving the straightforward modification in the case of two unary operations to the reader. Given a countably infinite set $X$, we define the set $T(X)$ of all unary semigroup terms over $X$ as follows:

- every $x \in X$ is a unary semigroup term and so is $x^{*}$;

- if $u$ and $v$ are unary semigroup terms, then so is their concatenation $u v$;

- if $u$ is a unary semigroup term, then so is $(u)^{*}$.

The algebraic structure $\mathcal{T}(X)=\left\langle T(X), \cdot,^{*}\right\rangle$ of type $(2,1)$ whose binary operation $\cdot$ is concatenation and whose unary operation is $u \mapsto(u)^{*}$ is called the free unary semigroup over $X$. By a unary semigroup identity over $X$ we mean a formal expression $u=v$ where $u, v \in T(X)$. A unary semigroup $\mathcal{S}=\left\langle S, \cdot,{ }^{*}\right\rangle$ satisfies the identity $u=v$ if the equality $\varphi(u)=\varphi(v)$ holds in $\mathcal{S}$ for all possible homomorphisms $\varphi: \mathcal{T}(X) \rightarrow \mathcal{S}$. Given $\mathcal{S}$, we denote by $\mathrm{Eq} \mathcal{S}$ the set of all unary semigroup identities it satisfies.

Given any collection $\Sigma$ of unary semigroup identities, we say that an identity $u=v$ follows from $\Sigma$ or that $\Sigma$ implies $u=v$ if every unary semigroup satisfying all identities of $\Sigma$ satisfies the identity $u=v$ as well. Birkhoff's completeness theorem of equational logic (see [5, Theorem 14.17]) shows that this notion (which we have given a semantic definition) can be captured by a very transparent set of inference rules. These rules in fact formalize the most natural things one does with identities: substitution of a term for a variable, application of operations to identities (such as, say, multiplying an identity through on the right by the same term) and using symmetry and transitivity of equality. We need not go into more detail here because the completeness theorem is not utilized in this paper.

Given a unary semigroup $\mathcal{S}$, an identity basis for $\mathcal{S}$ is any set $\Sigma \subseteq \mathrm{Eq} \mathcal{S}$ such that every identity of $\mathrm{Eq} \mathcal{S}$ follows from $\Sigma$. A unary semigroup $\mathcal{S}$ is said to be finitely based if it possesses a finite identity basis; otherwise $\mathcal{S}$ is called non-finitely based.

The class of all unary semigroups satisfying all identities from a given set $\Sigma$ of unary semigroup identities is called the variety defined by $\Sigma$. A variety is said to be finitely based if it can be defined by a finite set of identities; otherwise it is called non-finitely based. 
It is easy to see that the satisfaction of an identity is inherited by forming direct products and taking unary subsemigroups and homomorphic images, so that each variety is closed under these operators. In fact, varieties can be characterized by this closure property (the HSP-theorem, see [5, Theorem 11.9]). Given a unary semigroup $\mathcal{S}$, the variety defined by $\mathrm{Eq} \mathcal{S}$ is the variety generated by $\mathcal{S}$; we denote it by var $\mathcal{S}$. From the HSPtheorem it follows that every member of var $\mathcal{S}$ is a homomorphic image of a unary subsemigroup of a direct product of several copies of $\mathcal{S}$. Observe also that a unary semigroup and the variety it generates are simultaneously finitely or non-finitely based.

A variety $\mathbf{V}$ is said to be locally finite if every finitely generated member of $\mathbf{V}$ is finite. A finite unary semigroup is called inherently non-finitely based if it is not contained in any finitely based locally finite variety. Since the variety generated by a finite unary semigroup is locally finite (this is an easy consequence of the HSP-theorem, see [5, Theorem 10.16]), the property of being inherently non-finitely based implies the property of being non-finitely based; in fact, the former property is much stronger.

\subsection{Vocabulary of semigroup theory}

Since the algebraic structures we deal with are semigroups with some additional operation(s), we freely use the standard terminology and notation of semigroup theory, mostly following the early chapters of the textbook [6]. It should be noted, however, that our presentation is to a reasonable extent self-contained and does not require any specific semigroup-theoretic background.

In general, considering a unary semigroup $\mathcal{S}=\left\langle S, \cdot,{ }^{*}\right\rangle$, we do not assume any additional identities involving the unary operation *. If the identities $(x y)^{*}=y^{*} x^{*}$ and $\left(x^{*}\right)^{*}=x$ happen to hold in $\mathcal{S}$, in other words, if the unary operation $x \mapsto x^{*}$ is an involutory anti-automorphism of the semigroup $\langle S, \cdot\rangle$, we call $S$ an involutory semigroup. If, in addition, the identity $x=x x^{*} x$ holds, $\mathcal{S}$ is said to be a regular $*$-semigroup. Each group, subject to its inverse operation $x \mapsto x^{-1}$, is an involutory semigroup, even a regular *-semigroup; throughout the paper, any group is considered as a unary semigroup with respect to this inverse unary operation.

A wealth of examples of involutory semigroups and regular $*$-semigroups can be obtained via the following 'unary' version of the well known Rees matrix construction. ${ }^{2}$ Let $\mathcal{G}=\left\langle G, \cdot,^{-1}\right\rangle$ be a group, 0 a symbol beyond $G$, and $I$ a non-empty set. We formally set $0^{-1}=0$. Given an $I \times I$-matrix $P=\left(p_{i j}\right)$ over $G \cup\{0\}$ such that $p_{i j}=p_{j i}^{-1}$ for all $i, j \in I$, we define a multiplication - and a unary operation * on the set $(I \times G \times I) \cup\{0\}$ by the following rules:

$$
\begin{aligned}
& a \cdot 0=0 \cdot a=0 \quad \text { for all } a \in(I \times G \times I) \cup\{0\}, \\
& (i, g, j) \cdot(k, h, \ell)= \begin{cases}\left(i, g p_{j k} h, \ell\right) & \text { if } p_{j k} \neq 0, \\
0 & \text { if } p_{j k}=0,\end{cases} \\
& (i, g, j)^{*}=\left(j, g^{-1}, i\right), \quad 0^{*}=0 .
\end{aligned}
$$

\footnotetext{
2 See [6, Section 3.1] for a description of the construction in the plain semigroup case and [19, Section 2] for a detailed analysis of its unary version.
} 
It can be easily checked that $\left\langle(I \times G \times I) \cup\{0\}, \cdot{ }^{*}\right\rangle$ becomes an involutory semigroup; it will be a regular $*$-semigroup precisely when $p_{i i}=e$ (the identity element of the group $\mathcal{G}$ ) for all $i \in I$. We denote this unary semigroup by $\mathcal{M}^{0}(I, \mathcal{G}, I ; P)$ and call it the unary Rees matrix semigroup over $\mathcal{G}$ with the sandwich matrix $P$. If the group $\mathcal{G}$ involved happens to be the trivial group $\mathcal{E}=\{e\}$, then we shall ignore the group entry and represent the non-zero elements of such a Rees matrix semigroup by the pairs $(i, j)$ with $i, j \in I$.

In this paper, the 10-element unary Rees matrix semigroup over the trivial group $\mathcal{E}=\{e\}$ with the sandwich matrix

$$
\left(\begin{array}{lll}
e & e & e \\
e & e & 0 \\
e & 0 & e
\end{array}\right)
$$

plays a key role; we denote this semigroup by $\mathcal{K}_{3}$. Thus, subject to the convention mentioned above, $\mathcal{K}_{3}$ consists of the nine pairs $(i, j), i, j \in\{1,2,3\}$, and the element 0 , and the operations restricted to its non-zero elements can be described as follows:

$$
\begin{aligned}
(i, j) \cdot(k, \ell) & = \begin{cases}(i, \ell) & \text { if }(j, k) \neq(2,3),(3,2), \\
0 & \text { otherwise }\end{cases} \\
(i, j)^{*} & =(j, i) .
\end{aligned}
$$

Another unary semigroup that will be quite useful is the free involutory semigroup $\mathcal{F J}(X)$ on a given alphabet $X$. It can be constructed as follows. Let $\bar{X}=\left\{x^{*} \mid x \in X\right\}$ be a disjoint copy of $X$ and define $\left(x^{*}\right)^{*}=x$ for all $x^{*} \in \bar{X}$. Then $\mathcal{F J}(X)$ is the free semigroup $(X \cup \bar{X})^{+}$endowed with an involution * defined by

$$
\left(x_{1} \cdots x_{m}\right)^{*}=x_{m}^{*} \cdots x_{1}^{*}
$$

for all $x_{1}, \ldots, x_{m} \in X \cup \bar{X}$. See [19, Section 3] for more details on $\mathcal{F J}(X)$.

We will refer to elements of $\mathcal{F J}(X)$ as involutory words over $X$ while elements of the free semigroup $X^{+}$will be referred to as (plain semigroup) words over $X$.

\subsection{A property of matrices of rank 1}

Given a field $\mathcal{K}=\langle K,+, \cdot\rangle$, we denote the set of all $n \times n$-matrices over $\mathcal{K}$ by $\mathrm{M}_{n}(\mathcal{K})$. As mentioned in the introduction, in order to avoid trivialities, we always assume that $n \geq 2$.

We conclude our preparations by recording a simple property of rank 1 matrices. This property is, of course, known, but we do provide a proof for the sake of completeness.

Lemma 1.1. If $A \in \mathrm{M}_{n}(\mathcal{K})$ has rank 1 , then $A^{2} B A=A B A^{2}$ for any $B \in \mathrm{M}_{n}(\mathcal{K})$.

Proof. Consider the natural action of $A$ on the vector space $K^{n}$. The null-space $N(A)=$ $\left\{x \in K^{n} \mid x A=0\right\}$ has dimension $n-1$, whence the generalized eigenspace of $A$ corresponding to the eigenvalue 0 coincides with either $K^{n}$ or $N(A)$. In the former case $A^{2}=0$ and $A^{2} B A=0=A B A^{2}$ for any matrix $B$. In the latter case $K^{n}$ decomposes into the direct sum of $N(A)$ and the range $R(A)=\left\{x A \mid x \in K^{n}\right\}$ (see [37, Section 5.10]). Then $R(A)$ is in fact the (generalized) eigenspace of $A$ corresponding to a non-zero eigenvalue 
$\alpha \in K$ and the minimal polynomial of $A$ is $x(x-\alpha)$. Thus, $A$ satisfies the equation $A^{2}-\alpha A=0$, whence $A^{2} B A=\alpha A B A=A B \alpha A=A B A^{2}$, as required.

Let $\mathrm{L}_{n}(\mathcal{K})$ denote the set of all $n \times n$-matrices of rank at most 1 over $\mathcal{K}$. Adding the identity matrix to $\mathrm{L}_{n}(\mathcal{K})$ we get a set which we denote by $\mathrm{L}_{n}^{1}(\mathcal{K})$. Clearly, it is closed under matrix multiplication. From Lemma 1.1 we immediately obtain

Corollary 1.2. The semigroup $\left\langle\mathrm{L}_{n}^{1}(\mathcal{K}), \cdot\right\rangle$ satisfies the identity

$$
x^{2} y x=x y x^{2} .
$$

Observe that every group satisfying (1.2) is abelian.

\section{Tools}

\subsection{A unary version of the critical semigroup method}

Here we present a 'unary' modification of the approach used in [53]. According to the classification proposed in the survey [54], this approach is referred to as the critical semigroup method.

The formulation of the corresponding result involves two simple operators on unary semigroup varieties. For any unary semigroup $\mathcal{S}=\left\langle S, \cdot,{ }^{*}\right\rangle$ we denote by $\mathrm{H}(\mathcal{S})$ the unary subsemigroup of $\mathcal{S}$ which is generated by all elements of the form $x x^{*}$, where $x \in S$. We call $\mathrm{H}(\mathcal{S})$ the Hermitian subsemigroup of $\mathcal{S}$. For any variety $\mathbf{V}$ of unary semigroups, let $\mathrm{H}(\mathbf{V})$ be the subvariety of $\mathbf{V}$ generated by all Hermitian subsemigroups of members of $\mathbf{V}$. Likewise, given a positive integer $n$, let $\mathrm{P}_{n}(\mathcal{S})$ be the unary subsemigroup of $\mathcal{S}$ which is generated by all elements of the form $x^{n}$, where $x \in S$, and let $\mathrm{P}_{n}(\mathbf{V})$ be the subvariety of $\mathbf{V}$ generated by all subsemigroups $\mathrm{P}_{n}(\mathcal{S})$, where $\mathcal{S} \in \mathbf{V}$.

The following easy observation will be useful as it helps calculating the effect of the operators $\mathrm{H}$ and $\mathrm{P}_{n}$.

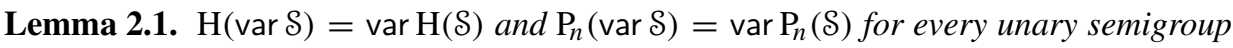
$\mathcal{S}$ and for each $n$.

Proof. The non-trivial part of the first claim is the inclusion $\mathrm{H}(\operatorname{var} \mathcal{S}) \subseteq \operatorname{var} \mathrm{H}(\mathcal{S})$. Let $\mathcal{T} \in \operatorname{var} \mathcal{S}$; then $\mathcal{T}$ is a homomorphic image of a unary subsemigroup $\mathcal{U}$ of a direct product of several copies of $\mathcal{S}$. But then $\mathrm{H}(\mathcal{T})$ is a homomorphic image of $\mathrm{H}(\mathcal{U})$. As is easy to see, $\mathrm{H}(\mathcal{U})$ is a unary subsemigroup of a direct product of several copies of $H(\mathcal{S})$. Thus $\mathrm{H}(\mathcal{T}) \in \operatorname{var} \mathrm{H}(\mathcal{S})$, and so $\mathrm{H}(\operatorname{var} \mathcal{S}) \subseteq \operatorname{var} \mathrm{H}(\mathcal{S})$. The second assertion can be treated in a completely similar way.

We are now ready to state the main result of this subsection.

Theorem 2.2. Let $\mathbf{V}$ be any unary semigroup variety such that $\mathcal{K}_{3} \in \mathbf{V}$. If either

- there exists a group $\mathcal{G}$ such that $\mathcal{G} \in \mathbf{V}$ but $\mathcal{G} \notin \mathrm{H}(\mathbf{V})$, or

- there exist a positive integer $d$ and a group $\mathcal{G}$ of exponent dividing $d$ such that $\mathcal{G} \in \mathbf{V}$ but $\mathcal{G} \notin \mathrm{P}_{d}(\mathbf{V})$,

then $\mathbf{V}$ has no finite basis of identities. 
Proof. Assume first that there exists a group $\mathcal{G} \in \mathbf{V} \backslash \mathrm{H}(\mathbf{V})$.

1. First we recall the basic idea of the 'critical semigroup method' in the unary setting. Suppose that $\mathbf{V}$ is finitely based. If $\Sigma$ is a finite identity basis of the variety $\mathbf{V}$ then there exists a positive integer $\ell$ such that all identities from $\Sigma$ depend on at most $\ell$ letters. Therefore the identities from $\Sigma$ hold in a unary semigroup $\mathcal{S}$ whenever all $\ell$-generated unary subsemigroups of $\mathcal{S}$ satisfy $\Sigma$. In other words, $\mathcal{S}$ belongs to $\mathbf{V}$ whenever all of its $\ell$-generated unary subsemigroups are in $\mathbf{V}$. We see that in order to prove our theorem it is sufficient to construct, for any given positive integer $k$, a unary semigroup $\mathcal{T}_{k} \notin \mathbf{V}$ for which all $k$-generated unary subsemigroups of $\mathcal{T}_{k}$ belong to $\mathbf{V}$.

2. Fix an identity $u\left(x_{1}, \ldots, x_{m}\right)=v\left(x_{1}, \ldots, x_{m}\right)$ that holds in $\mathrm{H}(\mathbf{V})$ but fails in the group $\mathcal{G}=\left\langle G, \cdot,{ }^{-1}\right\rangle$. The latter means that, for some $g_{1}, \ldots, g_{m} \in G$, substitution of $g_{i}$ for $x_{i}$ yields

$$
u\left(g_{1}, \ldots, g_{m}\right) \neq v\left(g_{1}, \ldots, g_{m}\right) .
$$

Now, for each positive integer $k$, let $n=\max \{4,2 k+1\}, I=\{1, \ldots, n m\}$ and consider the unary Rees matrix semigroup $\mathcal{T}_{k}=\mathcal{N}^{0}\left(I, \mathcal{G}, I ; P_{k}\right)$ over the group $\mathcal{G}$ with the sandwich matrix

$$
P_{k}=\left(\begin{array}{cccccc}
M_{n}\left(g_{1}\right) & E_{n} & O_{n} & \cdots & O_{n} & E_{n}^{T} \\
E_{n}^{T} & M_{n}\left(g_{2}\right) & E_{n} & \cdots & O_{n} & O_{n} \\
O_{n} & E_{n}^{T} & M_{n}\left(g_{3}\right) & \cdots & O_{n} & O_{n} \\
\vdots & \vdots & \vdots & \ddots & \vdots & \vdots \\
O_{n} & O_{n} & O_{n} & \cdots & M_{n}\left(g_{m-1}\right) & E_{n} \\
E_{n} & O_{n} & O_{n} & \cdots & E_{n}^{T} & M_{n}\left(g_{m}\right)
\end{array}\right),
$$

where $O_{n}$ is the zero $n \times n$-matrix, $E_{n}$ is the $n \times n$-matrix having $e$ (the identity of $\mathcal{G}$ ) in the position $(n, 1)$ and 0 in all other positions, $E_{n}^{T}$ is the transpose of $E_{n}$, and $M_{n}(g)$ denotes the $n \times n$-matrix

$$
M_{n}(g)=\left(\begin{array}{cccccc}
e & g & 0 & \cdots & 0 & e \\
g^{-1} & e & e & \cdots & 0 & 0 \\
0 & e & e & \cdots & 0 & 0 \\
\vdots & \vdots & \vdots & \ddots & \vdots & \vdots \\
0 & 0 & 0 & \cdots & e & e \\
e & 0 & 0 & \cdots & e & e
\end{array}\right) .
$$

(This construction is in a sense a combination of those of [3] and [53].) We aim to prove that $\mathcal{T}_{k}$ enjoys the two properties needed, namely, it does not belong to $\mathbf{V}$, but each $k$ generated unary subsemigroup of $\mathcal{T}_{k}$ lies in $\mathbf{V}$.

3. In order to prove that $\mathcal{T}_{k} \notin \mathbf{V}$, we construct an identity that holds in $\mathbf{V}$, but fails in $\mathcal{T}_{k}$. Consider the following $m$ terms in $m n$ letters $x_{1}, \ldots, x_{m n}$ (the square brackets in these terms serve only to improve readability):

$$
\begin{array}{r}
w_{1}=\left[x_{1} x_{1}^{*} \cdots x_{n} x_{n}^{*}\right]\left[\left(x_{n+1} \cdots x_{2 n}\right)\left(x_{n+1} \cdots x_{2 n}\right)^{*} x_{2 n} x_{2 n}^{*}\right] \cdots \\
{\left[\left(x_{(m-1) n+1} \cdots x_{m n}\right)\left(x_{(m-1) n+1} \cdots x_{m n}\right)^{*} x_{m n} x_{m n}^{*}\right],}
\end{array}
$$




$$
\begin{aligned}
& w_{2}=\left[\left(x_{1} \cdots x_{n}\right)\left(x_{1} \cdots x_{n}\right)^{*} x_{n} x_{n}^{*}\right]\left[x_{n+1} x_{n+1}^{*} \cdots x_{2 n} x_{2 n}^{*}\right] \times \\
& {\left[\left(x_{2 n+1} \cdots x_{3 n}\right)\left(x_{2 n+1} \cdots x_{3 n}\right)^{*} x_{3 n} x_{3 n}^{*}\right] \cdots} \\
& {\left[\left(x_{(m-1) n+1} \cdots x_{m n}\right)\left(x_{(m-1) n+1} \cdots x_{m n}\right)^{*} x_{m n} x_{m n}^{*}\right],} \\
& w_{3}=\left[\left(x_{1} \cdots x_{n}\right)\left(x_{1} \cdots x_{n}\right)^{*} x_{n} x_{n}^{*}\right]\left[\left(x_{n+1} \cdots x_{2 n}\right)\left(x_{n+1} \cdots x_{2 n}\right)^{*} x_{2 n} x_{2 n}^{*}\right] \times \\
& {\left[x_{2 n+1} x_{2 n+1}^{*} \cdots x_{3 n} x_{3 n}^{*}\right] \cdots\left[\left(x_{(m-1) n+1} \ldots x_{m n}\right)\left(x_{(m-1) n+1} \ldots x_{m n}\right)^{*} x_{m n} x_{m n}^{*}\right] \text {, }} \\
& w_{m}=\left[\left(x_{1} \cdots x_{n}\right)\left(x_{1} \cdots x_{n}\right)^{*} x_{n} x_{n}^{*}\right]\left[\left(x_{n+1} \cdots x_{2 n}\right)\left(x_{n+1} \cdots x_{2 n}\right)^{*} x_{2 n} x_{2 n}^{*}\right] \ldots \\
& {\left[\left(x_{(m-2) n+1} \cdots x_{(m-1) n}\right)\left(x_{(m-2) n+1} \cdots x_{(m-1) n}\right)^{*} x_{(m-1) n} x_{(m-1) n}^{*}\right] \times} \\
& {\left[x_{(m-1) n+1} x_{(m-1) n+1}^{*} \cdots x_{m n} x_{m n}^{*}\right] .}
\end{aligned}
$$

Substituting $w_{i}$ for $x_{i}$ in $u$ respectively $v$, we get the identity

$$
u\left(w_{1}, \ldots, w_{m}\right)=v\left(w_{1}, \ldots, w_{m}\right)
$$

which holds in the variety $\mathbf{V}$. Indeed, if we take any $\mathcal{S} \in \mathbf{V}$, then, since $s s^{*} \in \mathrm{H}(\mathcal{S})$ for any $s$ in $\mathcal{S}$, all the values of $w_{i}$ belong to the Hermitian subsemigroup $\mathrm{H}(\mathcal{S})$ of $\mathcal{S}$. This subsemigroup, however, lies in $\mathrm{H}(\mathbf{V})$, and therefore satisfies the identity $u=v$.

Now we shall show that (2.2) fails in $\mathcal{T}_{k}$. Indeed, substituting $(i, e, i) \in \mathcal{T}_{k}$ for $x_{i}$, we calculate that the value of every term of the form

$$
\left(x_{(j-1) n+1} \cdots x_{j n}\right)\left(x_{(j-1) n+1} \cdots x_{j n}\right)^{*} x_{j n} x_{j n}^{*}
$$

is $((j-1) n+1, e, j n)$ while the value of each term of the form

$$
x_{(j-1) n+1} x_{(j-1) n+1}^{*} \cdots x_{j n} x_{j n}^{*}
$$

is $\left((j-1) n+1, g_{j}, j n\right)$. Hence the value of $w_{j}$ is just $\left(1, g_{j}, m n\right)$. Therefore, under this substitution, the left hand side of (2.2) takes the value $\left(s, u\left(g_{1}, \ldots, g_{m}\right), t\right)$ for suitable $s, t \in\{1, m n\}$ while the value of the right hand side is $\left(s^{\prime}, v\left(g_{1}, \ldots, g_{m}\right), t^{\prime}\right)$ (again for suitable $\left.s^{\prime}, t^{\prime} \in\{1, m n\}\right)$. In view of (2.1), these elements do not coincide in $\mathcal{T}_{k}$.

4. It remains to prove that each $k$-generated unary subsemigroup of $\mathcal{T}_{k}$ lies in $\mathbf{V}$. For every $m$-tuple $\left(\lambda_{1}, \ldots, \lambda_{m}\right)$ of positive integers satisfying

$$
1 \leq \lambda_{1} \leq n<\lambda_{2} \leq 2 n<\lambda_{3} \leq \cdots \leq(m-1) n<\lambda_{m} \leq m n
$$

consider the unary subsemigroup $\mathcal{T}_{k}\left(\lambda_{1}, \ldots, \lambda_{m}\right)$ of $\mathcal{T}_{k}$ consisting of 0 and all triples $(i, g, j)$ such that $g \in G$ and $i, j \notin\left\{\lambda_{1}, \ldots, \lambda_{m}\right\}$. As $2 k<n$ according to our choice of $n$, one concludes that any given $k$ elements of $\mathcal{T}_{k}$ must be contained in $\mathcal{T}_{k}\left(\lambda_{1}, \ldots, \lambda_{m}\right)$ for suitable $\lambda_{1}, \ldots, \lambda_{m}$. Thus it is sufficient to prove that each semigroup of the form $\mathcal{T}_{k}\left(\lambda_{1}, \ldots, \lambda_{m}\right)$ belongs to the variety $\mathbf{V}$.

Let us fix positive integers $\lambda_{1}, \ldots, \lambda_{m}$ satisfying (2.3). When multiplying triples from $\mathcal{T}_{k}\left(\lambda_{1}, \ldots, \lambda_{m}\right)$, the $\lambda_{1}^{\text {th }}, \ldots, \lambda_{m}^{\text {th }}$ rows and columns of the sandwich matrix $P_{k}$ are never involved. Therefore we can identify $\mathcal{T}_{k}\left(\lambda_{1}, \ldots, \lambda_{m}\right)$ with the unary Rees matrix semigroup $\mathcal{M}^{0}\left(I^{\prime}, \mathcal{G}, I^{\prime} ; P_{k}^{\prime}\right)$ over the group $\mathcal{G}$ where $I^{\prime}=I \backslash\left\{\lambda_{1}, \ldots, \lambda_{m}\right\}$ and the sandwich matrix $P_{k}^{\prime}=P_{k}\left(\lambda_{1}, \ldots, \lambda_{m}\right)$ is obtained from $P_{k}$ by deleting its $\lambda_{1}^{\text {th }}, \ldots, \lambda_{m}^{\text {th }}$ rows and 
columns. Note that by (2.3) exactly one row and one column of each block $M_{n}\left(g_{i}\right)$ is deleted.

Now we transform the matrix $P_{k}\left(\lambda_{1}, \ldots, \lambda_{m}\right)$ as follows. For each $i$ with $(i-1) n+2$ $<\lambda_{i}$, we multiply successively

$$
\begin{aligned}
& \text { row }(i-1) n+2 \text { by } g_{i} \text { from the left and } \\
& \text { column }(i-1) n+2 \text { by } g_{i}^{-1} \text { from the right; } \\
& \text { row }(i-1) n+3 \text { by } g_{i} \text { from the left and } \\
& \text { column }(i-1) n+3 \text { by } g_{i}^{-1} \text { from the right; }
\end{aligned}
$$

row $\lambda_{i}-1$ by $g_{i}$ from the left and

column $\lambda_{i}-1$ by $g_{i}^{-1}$ from the right.

In order to help the reader to understand the effect of the transformations (2.4), we illustrate their action on the block obtained from $M_{n}\left(g_{i}\right)$ by removing the $\lambda_{i}^{\text {th }}$ row and column in the following scheme in which $\lambda_{i}$ has been chosen to be equal to $(i-1) n+5$. (The transformations have no effect beyond $M_{n}\left(g_{i}\right)$ because all the rows and columns of $P_{k}\left(\lambda_{1}, \ldots, \lambda_{m}\right)$ involved in (2.4) have non-zero entries only within $M_{n}\left(g_{i}\right)$.)

The block obtained from $M_{n}\left(g_{i}\right)$ by erasing the $((i-1) n+5)^{\text {th }}$ row and column

$$
\left(\begin{array}{cccccccc}
e & g_{i} & 0 & 0 & 0 & \cdots & 0 & e \\
g_{i}^{-1} & e & e & 0 & 0 & \cdots & 0 & 0 \\
0 & e & e & e & 0 & \cdots & 0 & 0 \\
0 & 0 & e & e & 0 & \cdots & 0 & 0 \\
0 & 0 & 0 & 0 & e & \cdots & 0 & 0 \\
\vdots & \vdots & \vdots & \vdots & \vdots & \ddots & \vdots & \vdots \\
0 & 0 & 0 & 0 & 0 & \cdots & e & e \\
e & 0 & 0 & 0 & 0 & \cdots & e & e
\end{array}\right)
$$

After the second transformation

$$
\left(\begin{array}{cccccccc}
e & e & 0 & 0 & 0 & \cdots & 0 & e \\
e & e & e & 0 & 0 & \cdots & 0 & 0 \\
0 & e & e & g_{i} & 0 & \cdots & 0 & 0 \\
0 & 0 & g_{i}^{-1} & e & 0 & \cdots & 0 & 0 \\
0 & 0 & 0 & 0 & e & \cdots & 0 & 0 \\
\vdots & \vdots & \vdots & \vdots & \vdots & \ddots & \vdots & \vdots \\
0 & 0 & 0 & 0 & 0 & \cdots & e & e \\
e & 0 & 0 & 0 & 0 & \cdots & e & e
\end{array}\right)
$$

After the first

transformation

$$
\left(\begin{array}{cccccccc}
e & e & 0 & 0 & 0 & \cdots & 0 & e \\
e & e & g_{i} & 0 & 0 & \cdots & 0 & 0 \\
0 & g_{i}^{-1} & e & e & 0 & \cdots & 0 & 0 \\
0 & 0 & e & e & 0 & \cdots & 0 & 0 \\
0 & 0 & 0 & 0 & e & \cdots & 0 & 0 \\
\vdots & \vdots & \vdots & \vdots & \vdots & \ddots & \vdots & \vdots \\
0 & 0 & 0 & 0 & 0 & \cdots & e & e \\
e & 0 & 0 & 0 & 0 & \cdots & e & e
\end{array}\right)
$$

After the third transformation

$$
\left(\begin{array}{cccccccc}
e & e & 0 & 0 & 0 & \cdots & 0 & e \\
e & e & e & 0 & 0 & \cdots & 0 & 0 \\
0 & e & e & e & 0 & \cdots & 0 & 0 \\
0 & 0 & e & e & 0 & \cdots & 0 & 0 \\
0 & 0 & 0 & 0 & e & \cdots & 0 & 0 \\
\vdots & \vdots & \vdots & \vdots & \vdots & \ddots & \vdots & \vdots \\
0 & 0 & 0 & 0 & 0 & \cdots & e & e \\
e & 0 & 0 & 0 & 0 & \cdots & e & e
\end{array}\right)
$$

Now it should be clear that also in general the transformations (2.4) result in a matrix $Q_{k}$ all of whose non-zero entries are equal to $e$. On the other hand, it is known (see, e.g., 
[3, Proposition 6.2]) that the transformations (2.4) of the sandwich matrix do not change the unary semigroup $\mathcal{T}_{k}\left(\lambda_{1}, \ldots, \lambda_{m}\right)$; in other words $\mathcal{T}_{k}\left(\lambda_{1}, \ldots, \lambda_{m}\right)$ is isomorphic to the $I^{\prime} \times I^{\prime}$ unary Rees matrix semigroup $\mathcal{R}_{k}$ over $\mathcal{G}$ with the sandwich matrix $Q_{k}$. Let $\mathcal{U}_{k}$ be the $I^{\prime} \times I^{\prime}$ unary Rees matrix semigroup over the trivial group $\mathcal{E}$ with the sandwich matrix $Q_{k}$. It is easy to check that the mapping $\mathcal{G} \times \mathcal{U}_{k} \rightarrow \mathcal{R}_{k}$ defined by

$$
(g,(i, j)) \mapsto(i, g, j), \quad(g, 0) \mapsto 0,
$$

for all $g \in G, i, j \in I^{\prime}$, is a unary semigroup homomorphism onto $\mathcal{R}_{k}$. Now we note that $\mathcal{G} \in \mathbf{V}$ and $\mathcal{U}_{k}$ belongs to the variety generated by $\mathcal{K}_{3}$ (see [3, Theorem 5.2]). This yields $\mathcal{T}_{k}\left(\lambda_{1}, \ldots, \lambda_{m}\right) \cong \mathcal{R}_{k} \in \mathbf{V}$.

The case when there exist a positive integer $d$ and a group $\mathcal{G}$ of exponent dividing $d$ such that $\mathcal{G} \in \mathbf{V} \backslash \mathrm{P}_{d}(\mathbf{V})$ can be treated in a very similar way. The construction of the critical semigroups remains the same, and the only modification to be made in the rest of the proof is to replace the terms $w_{i}$ above by the following plain semigroup words:

$$
\begin{aligned}
& w_{1}=\left[x_{1}^{d} \cdots x_{n}^{d}\right]\left[x_{n+1} \cdots x_{2 n}\right]^{d} \cdots\left[x_{(m-1) n+1} \cdots x_{m n}\right]^{d}, \\
& w_{2}=\left[x_{1} \cdots x_{n}\right]^{d}\left[x_{n+1}^{d} \cdots x_{2 n}^{d}\right] \cdots\left[x_{(m-1) n+1} \cdots x_{m n}\right]^{d}, \\
& w_{m}=\left[x_{1} \cdots x_{n}\right]^{d}\left[x_{n+1} \cdots x_{2 n}\right]^{d} \cdots\left[x_{(m-1) n+1}^{d} \cdots x_{m n}^{d}\right] .
\end{aligned}
$$

(These words have already been used in the plain semigroup case by the third author [53].)

\subsection{A unary version of the method of inherently non-finitely based semigroups}

Here we prove a sufficient condition for an involutory semigroup to be inherently nonfinitely based and exhibit two concrete examples of involutory semigroups satisfying this condition. These examples will be essential in our applications in Section 3.

Let $x_{1}, x_{2}, \ldots$ be a sequence of letters. The sequence $\left\{Z_{n}\right\}_{n=1,2, \ldots}$ of Zimin words is defined inductively by $Z_{1}=x_{1}, Z_{n+1}=Z_{n} x_{n+1} Z_{n}$. We say that an involutory word $v$ is an involutory isoterm for a unary semigroup $\mathcal{S}$ if the only involutory word $v^{\prime}$ such that $\mathcal{S}$ satisfies the involutory semigroup identity $v=v^{\prime}$ is the word $v$ itself.

Theorem 2.3. Let $\mathcal{S}$ be a finite involutory semigroup. If all Zimin words are involutory isoterms for $\mathcal{S}$, then $\mathcal{S}$ is inherently non-finitely based.

Proof. Aiming at a contradiction, suppose that $\mathcal{S}$ belongs to a finitely based locally finite variety $\mathbf{V}$. If $\Sigma$ is a finite identity basis of $\mathbf{V}$, then there exists a positive integer $\ell$ such that all identities from $\Sigma$ depend on at most $\ell$ letters. Clearly, all identities in $\Sigma$ hold in $\mathcal{S}$. In the following, our aim will be to construct, for any given positive integer $k$, an infinite, finitely generated involutory semigroup $\mathcal{T}_{k}$ which satisfies all identities in at most $k$ variables that hold in $\mathcal{S}$. In particular, $\mathcal{T}_{\ell}$ will satisfy all identities from $\Sigma$. This yields a contradiction, as then we must conclude that $\mathcal{T}_{\ell} \in \mathbf{V}$, which is impossible by the local finiteness of $\mathbf{V}$. 


$$
M=\left(\begin{array}{ccccc}
1 & 1 & \cdots & 1 & 1 \\
\vdots & \vdots & \ddots & \vdots & \vdots \\
1 & r & \cdots & 1 & r \\
2 & 1 & \cdots & 2 & 1 \\
\vdots & \vdots & \ddots & \vdots & \vdots \\
2 & r & \cdots & 2 & r \\
\vdots & \vdots & \ddots & \vdots & \vdots \\
r & 1 & \cdots & r & 1 \\
\vdots & \vdots & \ddots & \vdots & \vdots \\
r & r & \cdots & r & r
\end{array}\right) \quad M_{A}=\left(\begin{array}{ccccc}
a_{11} & a_{12} & \cdots & a_{1 r-1} & a_{1 r} \\
\vdots & \vdots & \ddots & \vdots & \vdots \\
a_{11} & a_{r 2} & \cdots & a_{1 r-1} & a_{r r} \\
a_{21} & a_{12} & \cdots & a_{2 r-1} & a_{1 r} \\
\vdots & \vdots & \ddots & \vdots & \vdots \\
a_{21} & a_{r 2} & \cdots & a_{2 r-1} & a_{r r} \\
\vdots & \vdots & \ddots & \vdots & \vdots \\
a_{r 1} & a_{12} & \cdots & a_{r r-1} & a_{1 r} \\
\vdots & \vdots & \ddots & \vdots & \vdots \\
a_{r 1} & a_{r 2} & \cdots & a_{r r-1} & a_{r r}
\end{array}\right)
$$

Fig. 1. The matrices $M$ and $M_{A}$

We shall employ a construction invented by Sapir [48] (see also his lecture notes [50]). We fix $k$ and let $r=6 k+2$. Consider the $r^{2} \times r$-matrix $M$ in Fig. 1 (left). All oddnumbered columns of $M$ are identical and equal to the transpose of the row $(1, \ldots, 1$, $2, \ldots, 2, \ldots, r, \ldots, r)$ where each number occurs $r$ times. All even-numbered columns of $M$ are identical and equal to the transpose of $(1,2, \ldots, r, \ldots, 1,2, \ldots, r)$ where the block $1,2, \ldots, r$ occurs $r$ times.

Now consider the alphabet $A=\left\{a_{i j} \mid 1 \leq i, j \leq r\right\}$ of cardinality $r^{2}$. We convert the matrix $M$ into the matrix $M_{A}$ (shown in Fig. 1, right) by replacing numbers by letters according to the following rule: whenever a number $i$ occurs in column $j$ of $M$, we substitute it with the letter $a_{i j}$ to get the corresponding entry in $M_{A}$.

Let $v_{t}$ be the word in the $t^{\text {th }}$ row of $M_{A}$. Consider the endomorphism $\gamma: A^{+} \rightarrow A^{+}$ defined by

$$
\gamma\left(a_{i j}\right)=v_{(i-1) r+j} .
$$

Let $V_{k}$ be the set of all factors of the words in the sequence $\left\{\gamma^{m}\left(a_{11}\right)\right\}_{m=1,2, \ldots}$ and let 0 be a symbol beyond $V_{k}$. We define a multiplication . on the set $V_{k} \cup\{0\}$ as follows:

$$
u \cdot v= \begin{cases}u v & \text { if } u, v, u v \in V_{k}, \\ 0 & \text { otherwise. }\end{cases}
$$

Clearly, $\left\langle V_{k} \cup\{0\}, \cdot\right\rangle$ becomes a semigroup which we denote by $\mathcal{V}_{k}^{0}$. Using this semigroup, we can conveniently reformulate two major combinatorial results by Sapir:

Proposition 2.4 ([50, Proposition 2.1]). Let $X_{k}=\left\{x_{1}, \ldots, x_{k}\right\}$ and $w \in X_{k}^{+}$. Assume that there exists a homomorphism $\varphi: X_{k}^{+} \rightarrow V_{k}^{0}$ for which $\varphi(w) \neq 0$. Then there is an endomorphism $\psi: X_{k}^{+} \rightarrow X_{k}^{+}$such that the word $\psi(w)$ appears as a factor in the Zimin word $Z_{k}$.

Proposition 2.5 ([50, Lemma 4.14]). Let $X_{k}=\left\{x_{1}, \ldots, x_{k}\right\}$ and $w, w^{\prime} \in X_{k}^{+}$. Assume that there exists a homomorphism $\varphi: X_{k}^{+} \rightarrow V_{k}^{0}$ for which $\varphi(w) \neq \varphi\left(w^{\prime}\right)$. Then the identity $w=w^{\prime}$ implies a non-trivial semigroup identity of the form $Z_{k+1}=z$. 
Now let $\overline{\mathcal{V}}_{k}^{0}$ denote the semigroup anti-isomorphic to $\mathcal{V}_{k}^{0}$; we shall use the notation $x \mapsto x^{*}$ for the mutual anti-isomorphisms between $\mathcal{V}_{k}^{0}$ and $\overline{\mathcal{V}}_{k}^{0}$ in both directions and denote $\left\{v^{*} \mid\right.$ $\left.v \in V_{k}\right\}$ by $\bar{V}_{k}$. Let

$$
\mathcal{T}_{k}=\left\langle V_{k} \cup \bar{V}_{k} \cup\{0\}, \cdot{ }^{*}\right\rangle
$$

be the 0 -direct union of $\mathcal{V}_{k}^{0}$ and $\overline{\mathcal{V}}_{k}^{0}$; this means that we identify 0 with $0^{*}$, preserve the multiplication in both $\mathcal{V}_{k}^{0}$ and $\bar{V}_{k}^{0}$, and set $u \cdot v^{*}=u^{*} \cdot v=0$ for all $u, v \in V_{k}$. This is the unary semigroup we need.

It is clear that $\mathcal{T}_{k}$ is infinite and is generated (as a unary semigroup) by the set $A$ which is finite. It remains to verify that $\mathcal{T}_{k}$ satisfies every identity in at most $k$ variables that holds in our initial unary semigroup $\mathcal{S}$. So, let $p, q \in \mathcal{F} \mathcal{J}\left(X_{k}\right)$ and suppose that the identity $p=q$ holds in $\mathcal{S}$ but fails in $\mathcal{T}_{k}$. Then there exists a unary semigroup homomorphism $\varphi: \mathcal{F J}\left(X_{k}\right) \rightarrow \mathcal{T}_{k}$ for which $\varphi(p) \neq \varphi(q)$. Hence, at least one of the elements $\varphi(p)$ and $\varphi(q)$ is not 0 ; say $\varphi(p) \neq 0$. Then we may also assume $\varphi(p) \in V_{k}$; otherwise we may consider the identity $p^{*}=q^{*}$ instead of $p=q$. Since $\varphi(p) \neq 0$, there is no letter $x \in X_{k}$ such that $p$ contains both $x$ and $x^{*}$. Now we define a substitution $\sigma: \mathcal{F J}\left(X_{k}\right) \rightarrow \mathcal{F J}\left(X_{k}\right)$ as follows:

$$
\sigma(x)= \begin{cases}x^{*} & \text { if } p \text { contains } x^{*} \\ x & \text { otherwise. }\end{cases}
$$

Then $\sigma(p)$ does not contain any starred letter, thus being a plain word in $X_{k}^{+}$. Since $\sigma^{2}$ is the identity mapping, we have $\varphi(p)=(\varphi \sigma)(\sigma(p))$, and $\varphi \sigma$ maps $X_{k}^{+}$into $\mathcal{V}_{k}^{0}$. Now we consider two cases.

Case 1: $\sigma(q)$ contains a starred letter. We apply Proposition 2.4 to the plain word $\sigma(p)$ and the semigroup homomorphism $X_{k}^{+} \rightarrow \mathcal{V}_{k}^{0}$ obtained by restricting $\varphi \sigma$ to $X_{k}^{+}$. We conclude that there is an endomorphism $\psi$ of $X_{k}^{+}$such that the word $\psi(\sigma(p))$ appears as a factor in the Zimin word $Z_{k}$. Thus, $Z_{k}=z^{\prime} \psi(\sigma(p)) z^{\prime \prime}$ for some $z^{\prime}, z^{\prime \prime}$ (which may be empty). The endomorphism $\psi$ extends in a natural way to an endomorphism of the free involutory semigroup $\mathcal{F J}\left(X_{k}\right)$ and there is no harm in denoting the extension by $\psi$ as well. The identity $p=q$ implies the identity

$$
z^{\prime} \psi(\sigma(p)) z^{\prime \prime}=z^{\prime} \psi(\sigma(q)) z^{\prime \prime} .
$$

The left hand side of (2.5) is $Z_{k}$ and the identity is not trivial because its right hand side involves a starred letter. Since $p=q$ holds in our initial semigroup $\mathcal{S}$, so does (2.5). But this contradicts the assumption that all Zimin words are involutory isoterms for $\mathcal{S}$.

Case 2: $\sigma(q)$ contains no starred letter. In this case $\sigma(q)$ is a plain word in $X_{k}^{+}$, and we are in a position to apply Proposition 2.5 to the semigroup identity $\sigma(p)=\sigma(q)$ and the semigroup homomorphism $X_{k}^{+} \rightarrow V_{k}^{0}$ obtained by restricting $\varphi \sigma$ to $X_{k}^{+}$. We conclude that $\sigma(p)=\sigma(q)$ implies a non-trivial semigroup identity $Z_{k+1}=z$. Therefore the identity $p=q$ implies $Z_{k+1}=z$, and we again get a contradiction.

Before passing to concrete examples of inherently non-finitely based involutory semigroups, we formulate a corollary of our proof of Theorem 2.3 which will be useful for 
our subsequent paper(s). Proposition 2.4 easily implies that no word in the sequence $\left\{\gamma^{m}\left(a_{11}\right)\right\}_{m=1,2, \ldots}$ has any square (that is, a word of the form $w w$ ) as a factor. Hence, the semigroup $\mathcal{V}_{k}^{0}$ satisfies the identity ${ }^{3} x^{2}=0$. This identity is clearly inherited by the involutory semigroup $\mathcal{T}_{k}$ which by its construction satisfies also the identity $x x^{*}=0$. Since $\mathcal{T}_{k}$ is finitely generated and infinite, we arrive at the following conclusion:

Corollary 2.6. If a variety $\mathbf{V}$ of involutory semigroups satisfies no non-trivial identity of the form $Z_{k+1}=z$ and all members of $\mathbf{V}$ satisfying the identities $x x^{*}=x^{2}=0$ are locally finite, then $\mathbf{V}$ is not finitely based.

This result is parallel to [48, Proposition 3] in the plain semigroup case.

Now consider the twisted Brandt monoid $\mathcal{T B}_{2}^{1}=\left\langle B_{2}^{1}, \cdot,{ }^{*}\right\rangle$, where $B_{2}^{1}$ is the set of the following six $2 \times 2$-matrices:

$$
\left(\begin{array}{ll}
0 & 0 \\
0 & 0
\end{array}\right), \quad\left(\begin{array}{ll}
1 & 0 \\
0 & 0
\end{array}\right), \quad\left(\begin{array}{ll}
0 & 1 \\
0 & 0
\end{array}\right), \quad\left(\begin{array}{ll}
0 & 0 \\
1 & 0
\end{array}\right), \quad\left(\begin{array}{ll}
0 & 0 \\
0 & 1
\end{array}\right),\left(\begin{array}{ll}
1 & 0 \\
0 & 1
\end{array}\right),
$$

the binary operation - is the usual matrix multiplication and the unary operation * fixes the matrices

$$
\left(\begin{array}{ll}
0 & 0 \\
0 & 0
\end{array}\right), \quad\left(\begin{array}{ll}
0 & 1 \\
0 & 0
\end{array}\right), \quad\left(\begin{array}{ll}
0 & 0 \\
1 & 0
\end{array}\right), \quad\left(\begin{array}{ll}
1 & 0 \\
0 & 1
\end{array}\right)
$$

and swaps each of the matrices

$$
\left(\begin{array}{ll}
1 & 0 \\
0 & 0
\end{array}\right), \quad\left(\begin{array}{ll}
0 & 0 \\
0 & 1
\end{array}\right)
$$

with the other one.

Corollary 2.7. The twisted Brandt monoid $\mathcal{T B}_{2}^{1}$ is inherently non-finitely based.

Proof. By Theorem 2.3 we only have to show that $\mathcal{T B}_{2}^{1}$ satisfies no non-trivial involutory semigroup identity of the form $Z_{n}=z$. If $z$ is a plain semigroup word, we can refer to [48, Lemma 3.7] which shows that the semigroup $\left\langle B_{2}^{1}, \cdot\right\rangle$ does not satisfy any non-trivial semigroup identity of the form $Z_{n}=z$. If we suppose that the involutory word $z$ contains a starred letter, we can substitute the matrix $\left(\begin{array}{ll}1 & 0 \\ 0 & 0\end{array}\right)$ for all letters occurring in $Z_{n}$ and $z$. Since this matrix is idempotent, the value of the word $Z_{n}$ under this substitution equals $\left(\begin{array}{ll}1 & 0 \\ 0 & 0\end{array}\right)$. On the other hand, $z$ evaluates to a product involving the matrix $\left(\begin{array}{ll}1 & 0 \\ 0 & 0\end{array}\right)^{*}=\left(\begin{array}{ll}0 & 0 \\ 0 & 1\end{array}\right)$, and it is easy to see that such a product is equal to either $\left(\begin{array}{ll}0 & 0 \\ 0 & 1\end{array}\right)$ or $\left(\begin{array}{ll}0 & 0 \\ 0 & 0\end{array}\right)$. Thus, the identity $Z_{n}=z$ cannot hold in $\mathcal{T B}_{2}^{1}$ in this case either.

An equivalent way to define $\mathcal{T B}_{2}^{1}$ is to consider the 5-element unary Rees matrix semigroup over the trivial group $\mathcal{E}=\{e\}$ with the sandwich matrix

$$
\left(\begin{array}{ll}
0 & e \\
e & 0
\end{array}\right)
$$

\footnotetext{
3 Strictly speaking the expression that follows is not an identity as defined before (since 0 is not a term) but rather an abbreviation for the identities $x^{2} y=x^{2}=y x^{2}$. However, referring to such abbreviations as identities is a standard convention which we adopt.
} 
and then to adjoin to this unary Rees matrix semigroup an identity element. For convenience and later use we note that $\mathcal{T B}_{2}^{1}$ can thus be realized as the set

$$
\{(1,1),(1,2),(2,1),(2,2), 0,1\}
$$

endowed with the operations

$$
\begin{gathered}
(i, j) \cdot(k, \ell)= \begin{cases}(i, \ell) & \text { if }(j, k) \in\{(1,2),(2,1)\}, \\
0 & \text { otherwise } ;\end{cases} \\
1 \cdot x=x=x \cdot 1, \quad 0 \cdot x=0=x \cdot 0 \quad \text { for all } x ; \\
(i, j)^{*}=(j, i), \quad 1^{*}=1, \quad 0^{*}=0 .
\end{gathered}
$$

Suppose that $\mathcal{S}$ is a finite unary semigroup for which the variety var $\mathcal{S}$ contains an inherently non-finitely based finite unary semigroup. Immediately from the definition it follows that $\mathcal{S}$ is also inherently non-finitely based. This observation is useful, in particular, for the justification of our second example of an involutory inherently non-finitely based semigroup. This is a 'twisted version' $\mathcal{T A}_{2}^{1}$ of another 6-element semigroup that often shows up under the name $A_{2}^{1}$ in the theory of semigroup varieties. The unary semigroup $\mathcal{T A}_{2}^{1}$ is formed by the six matrices

$$
\left(\begin{array}{ll}
0 & 0 \\
0 & 0
\end{array}\right), \quad\left(\begin{array}{ll}
1 & 0 \\
0 & 0
\end{array}\right), \quad\left(\begin{array}{ll}
0 & 1 \\
0 & 0
\end{array}\right), \quad\left(\begin{array}{ll}
1 & 0 \\
1 & 0
\end{array}\right), \quad\left(\begin{array}{ll}
0 & 1 \\
0 & 1
\end{array}\right), \quad\left(\begin{array}{ll}
1 & 0 \\
0 & 1
\end{array}\right)
$$

under the usual matrix multiplication and the unary operation that swaps each of the matrices

$$
\left(\begin{array}{ll}
1 & 0 \\
0 & 0
\end{array}\right), \quad\left(\begin{array}{ll}
0 & 1 \\
0 & 1
\end{array}\right)
$$

with the other one and fixes all other matrices. Alternatively, $\mathcal{T A}_{2}^{1}$ is obtained from the 5-element unary Rees matrix semigroup $\mathcal{A}_{2}$ over $\mathcal{E}=\{e\}$ with the sandwich matrix

$$
\left(\begin{array}{ll}
0 & e \\
e & e
\end{array}\right)
$$

by adjoining an identity element. Again, for later use, we note that $\mathcal{T A}_{2}^{1}$ can be realized as the set

$$
\{(1,1),(1,2),(2,1),(2,2), 0,1\}
$$

endowed with the operations

$$
\begin{gathered}
(i, j) \cdot(k, \ell)= \begin{cases}(i, \ell) & \text { if }(j, k) \neq(1,1), \\
0 & \text { if }(j, k)=(1,1) ;\end{cases} \\
1 \cdot x=x=x \cdot 1, \quad 0 \cdot x=0=x \cdot 0 \quad \text { for all } x ; \\
(i, j)^{*}=(j, i), \quad 1^{*}=1, \quad 0^{*}=0 .
\end{gathered}
$$

Corollary 2.8. The involutory semigroup $\mathcal{T A}_{2}^{1}$ is inherently non-finitely based. 
Proof. We represent $\mathcal{T A}_{2}^{1}$ as in (2.8) and $\mathcal{T B}_{2}^{1}$ as in (2.6) and consider the direct square $\mathcal{T A}_{2}^{1} \times \mathcal{T} \mathcal{A}_{2}^{1}$. It is then easy to check that the twisted Brandt monoid $\mathcal{T B}_{2}^{1}$ is a homomorphic image of the unary subsemigroup of $\mathcal{T A}_{2}^{1} \times \mathcal{T} \mathcal{A}_{2}^{1}$ generated by the pairs $(1,1)$, $((1,1),(2,2))$ and $((2,2),(1,1))$. Thus, $\mathcal{T B}_{2}^{1}$ belongs to var $\mathcal{T A}_{2}^{1}$. Since by Corollary 2.7 , $\mathcal{T B}_{2}^{1}$ is inherently non-finitely based, so is $\mathcal{T A}_{2}^{1}$.

Sapir [48, Proposition 7] has shown that a (plain) finite semigroup $\mathcal{S}$ is inherently nonfinitely based if and only if all Zimin words are isoterms for $\mathcal{S}$, that is, $\mathcal{S}$ satisfies no non-trivial semigroup identity of the form $Z_{n}=z$. Our Theorem 2.3 models the 'if' part of this statement but we do not know whether or not the 'only if' part transfers to the involutory environment. Some partial results in this direction have recently been obtained by the second author [11]. Here we present yet another special result which however suffices for our purposes.

Proposition 2.9. Let $\mathcal{S}=\left\langle S, \cdot,{ }^{*}\right\rangle$ be a finite involutory semigroup and suppose that there exists an involutory word $\omega(x)$ in one variable $x$ such that $\mathcal{S}$ satisfies the identity $x=x \omega(x) x$. Then $\mathcal{S}$ is not inherently non-finitely based.

Proof. We have to construct a finite set of identities that defines a locally finite variety of involutory semigroups containing $\mathcal{S}$. The crucial step towards this goal consists in verifying that the identity $x=x \omega(x) x$ allows one to express right divisibility in terms of equational logic. This being done, we shall be in a position to closely follow powerful arguments developed by Margolis and Sapir in [36].

We say that elements $a, b \in S$ divide each other on the right and write $a \mathscr{R} b$ if either $a=b$ or there exist $s, t \in S$ such that $a=b s$ and $b=a t$. Clearly, $\mathscr{R}$ is an equivalence relation (known as the right Green relation in semigroup theory).

Since $S$ satisfies the identity $x=x \omega(x) x$, we have $a \mathscr{R} a \omega(a)$ for each $a \in S$. (Indeed, $a=a \omega(a) \cdot a$ and $a \omega(a)=a \cdot \omega(a)$.) Thus, for $a, b \in S$, we have $a \mathscr{R} b$ if and only if $a \omega(a) \mathscr{R} b \omega(b)$. Since $a \omega(a)$ and $b \omega(b)$ are idempotents, that latter condition is equivalent to the two equalities $a \omega(a) \cdot b \omega(b)=b \omega(b)$ and $b \omega(b) \cdot a \omega(a)=a \omega(a)$. In particular, for $u, v \in S$ we have $u v \mathscr{R} u$ if and only if $u v \omega(u v) \cdot u \omega(u)=u \omega(u)$ (since the second equality $u \omega(u) \cdot u v \omega(u v)=u v \omega(u v)$ is always true).

Let $Z_{n}^{\prime}$ be the word obtained from the Zimin word $Z_{n}$ by deleting the last letter (which is $x_{1}$ ), that is, $Z_{n}^{\prime} x_{1}=Z_{n}$. Now set $n=|S|+1$; Lemma 7 in [36] implies that under every evaluation of the letters $x_{1}, \ldots, x_{n}$ in $\mathcal{S}$, the values of the words $Z_{n}^{\prime}$ and $Z_{n}$ divide each other on the right. As explained in the previous paragraph, this can be restated as saying that the values of the terms $Z_{n} \omega\left(Z_{n}\right) \cdot Z_{n}^{\prime} \omega\left(Z_{n}^{\prime}\right)$ and $Z_{n}^{\prime} \omega\left(Z_{n}^{\prime}\right)$ coincide under every evaluation of $x_{1}, \ldots, x_{n}$ in $\mathcal{S}$, that is, $\mathcal{S}$ satisfies the identity

$$
Z_{n} \omega\left(Z_{n}\right) \cdot Z_{n}^{\prime} \omega\left(Z_{n}^{\prime}\right)=Z_{n}^{\prime} \omega\left(Z_{n}^{\prime}\right)
$$

On the other hand, in each involutory semigroup $\mathcal{T}=\left\langle T, \cdot,{ }^{*}\right\rangle$ which satisfies $x=x \omega(x) x$ and (2.9), the values of the words $Z_{n}^{\prime}$ and $Z_{n}$ under every evaluation of $x_{1}, \ldots, x_{n}$ necessarily divide each other on the right. This implies that such a $\mathcal{T}$ satisfies the implication

$$
x Z_{n}=y Z_{n} \rightarrow x Z_{n}^{\prime}=y Z_{n}^{\prime} .
$$


Indeed, suppose that under some evaluation $\varphi$ of the letters $x, y, x_{1}, \ldots, x_{n}$ in $\mathcal{T}$, the words $x Z_{n}$ and $y Z_{n}$ happen to take the same value, that is, $\varphi\left(x Z_{n}\right)=\varphi\left(y Z_{n}\right)$. Since $\varphi\left(Z_{n}\right) \mathscr{R} \varphi\left(Z_{n}^{\prime}\right)$, there exists $t \in T$ such that $\varphi\left(Z_{n}\right) t=\varphi\left(Z_{n}^{\prime}\right)$. Hence

$$
\varphi\left(x Z_{n}^{\prime}\right)=\varphi\left(x Z_{n}\right) t=\varphi\left(y Z_{n}\right) t=\varphi\left(y Z_{n}^{\prime}\right),
$$

that is, the words $x Z_{n}^{\prime}$ and $y Z_{n}^{\prime}$ also take a common value under $\varphi$.

Lemma 8 in [36] shows that a finitely generated semigroup satisfying (2.10) is finite if and only if it satisfies the identity

$$
x^{k}=x^{k+\ell}
$$

for some $k, \ell \geq 1$ and has only locally finite subgroups. We note that an involutory semigroup $\mathcal{T}=\left\langle T, \cdot,{ }^{*}\right\rangle$ is finitely generated if and only if so is the semigroup $\langle T, \cdot\rangle$. An identity of the form (2.11) definitely holds in $\mathcal{S}$ since $\mathcal{S}$ is finite. Hence it suffices to find a finite number of identities which hold in $\mathcal{S}$ and which force each (involutory) semigroup to have only locally finite subgroups.

We can proceed as at the end of [36]. Let $\mathcal{G}$ be the direct product of all maximal subgroups of $\mathcal{S}$. By the Oates-Powell theorem [40] (see also [39, §5.2]), the locally finite variety $\operatorname{var} \mathcal{G}$ generated by the finite group $\mathcal{G}$ can be defined by a single identity $v\left(x_{1}, \ldots, x_{m}\right)=1$. The left hand side $v$ of this identity can be assumed to contain no occurrence of the inversion ${ }^{-1}$, that is, we may assume that $v$ is a plain semigroup word in the letters $x_{1}, \ldots, x_{m}$. Now let $\mathcal{F}=\mathcal{F}\left(x_{1}, \ldots, x_{m}\right)$ be the $m$-generated relatively free semigroup in the (locally finite) semigroup variety generated by the semigroup $\langle S, \cdot\rangle$. The semigroup $\mathcal{F}$ is finite and hence has a least ideal; this ideal is known to be a union of (isomorphic) subgroups (Sushkevich's theorem, see [6]). Let $\mathcal{H}$ be any of these subgroups and let $e$ be the identity element of $\mathcal{H}$. We denote by $u\left(x_{1}, \ldots, x_{m}\right)$ a word whose value in $\mathcal{F}$ is $e$. Since $e^{2}=e$, we see that $\mathcal{F}$ (and therefore $\mathcal{S}$ ) satisfies the identity

$$
u=u^{2} .
$$

For every $g \in \mathcal{F}$, the product ege belongs to $\mathcal{H}$. As observed in [27], the group $\mathcal{H}$ belongs to the variety var $\mathcal{G}$. Consequently, $\mathcal{F}$ (and therefore $\mathcal{S}$ ) satisfies the identity

$$
v\left(u x_{1} u, \ldots, u x_{m} u\right)=u .
$$

Note that both sides of (2.13) are plain semigroup words in the letters $x_{1}, \ldots, x_{m}$.

Now consider the variety $\mathbf{V}$ of involutory semigroups defined by the identity $x=$ $x \omega(x) x$, an identity of the form (2.11) holding in $\delta$, and the identities (2.9), (2.12), and (2.13). Since by the construction $\mathcal{S}$ satisfies all the listed identities, $\mathcal{S}$ is a member of $\mathbf{V}$. Let $\mathcal{T}=\left\langle T, \cdot,{ }^{*}\right\rangle$ be any finitely generated member of $\mathbf{V}$; then, as already mentioned, the semigroup $\langle T, \cdot\rangle$ is also finitely generated. The first and the third identity ensure that the semigroup $\langle T, \cdot\rangle$ satisfies the implication (2.10), and therefore it is finite provided that all its subgroups are locally finite. Each group that satisfies the identities (2.12) and (2.13) satisfies the identity $v\left(x_{1}, \ldots, x_{m}\right)=1$, hence belongs to $\operatorname{var} \mathcal{G}$ and so is locally finite. Altogether, $\mathcal{T}$ is finite. Thus, $\mathbf{V}$ is locally finite and finitely based, and the proposition is proved. 
Proposition 2.9 implies in particular that no finite regular $*$-semigroup can be inherently non-finitely based as one can use $x^{*}$ in the role of the term $\omega(x)$. In particular, the unary semigroup $\left\langle B_{2}^{1}, \cdot,{ }^{T}\right\rangle$, where the unary operation is the usual matrix transposition, is not inherently non-finitely based (this fact was first discovered by Sapir [49]), even though it is not finitely based [28].

\section{Applications}

\subsection{Matrix semigroups with Moore-Penrose inverse}

Certainly, the most common unary operation for matrices is transposition. However, it is convenient for us to start by analyzing matrix semigroups with Moore-Penrose inverse because this analysis will help us in considering semigroups with transposition.

We first recall the notion of Moore-Penrose inverse. This has been discovered by Moore [38] and independently by Penrose [41] for complex matrices, but has turned out to be a fruitful concept in a more general setting — see [4] for a comprehensive treatment.

The following results were obtained by Drazin [12].

Proposition 3.1 ([12, Proposition 1]). Let $\mathcal{S}$ be an involutory semigroup. Then, for any given $a \in \mathcal{S}$, the four equations

$$
\text { axa }=a, \quad x a x=x, \quad(a x)^{*}=a x, \quad(x a)^{*}=x a
$$

have at most one common solution $x \in \mathcal{S}$.

For an element $a$ of an involutory semigroup $\mathcal{S}$, we denote by $a^{\dagger}$ the unique common solution $x$ of the equations (3.1), provided it exists, and call $a^{\dagger}$ the Moore-Penrose inverse of $a$.

Recall that an element $a \in \mathcal{S}$ is said to be regular if there is an $x \in \mathcal{S}$ such that $a x a=a$. Concerning existence of the Moore-Penrose inverse, we have the following

Proposition 3.2 ([12, Proposition 2]). Let $\mathcal{S}$ be an involutory semigroup satisfying the implication

$$
x^{*} x=x^{*} y=y^{*} x=y^{*} y \rightarrow x=y .
$$

Then for $a \in \mathcal{S}$, the Moore-Penrose inverse $a^{\dagger}$ exists if and only if $a^{*} a$ and $a a^{*}$ are regular elements.

Let $\langle R,+, \cdot\rangle$ be a ring. A ring involution is an involution $x \mapsto x^{*}$ of the semigroup $\langle R, \cdot\rangle$ satisfying in addition the identity $(x+y)^{*}=x^{*}+y^{*}$. For ring involutions, the implication (3.2) is easily seen to be equivalent to

$$
x^{*} x=0 \rightarrow x=0
$$

Now suppose that $\mathcal{K}=\langle K,+, \cdot\rangle$ is a field that admits a ring involution $x \mapsto \bar{x}$. Then the matrix ring $\mathrm{M}_{n}(\mathcal{K})$ has an involution that naturally arises from the involution of $\mathcal{K}$, 
namely $\left(a_{i j}\right) \mapsto\left(a_{i j}\right)^{*}:=\left(\overline{a_{i j}}\right)^{T}$. This involution of $\mathrm{M}_{n}(\mathcal{K})$ in general does not satisfy the implication (3.3). However, it does satisfy (3.3) if and only if the equation

$$
x_{1} \overline{x_{1}}+x_{2} \overline{x_{2}}+\cdots+x_{n} \overline{x_{n}}=0
$$

admits only the trivial solution $\left(x_{1}, \ldots, x_{n}\right)=(0, \ldots, 0)$ in $K^{n}$. Since all elements of $\mathbf{M}_{n}(\mathcal{K})$ are regular, this means that the Moore-Penrose inverse exists-subject to the involution $\left(a_{i j}\right) \mapsto\left(a_{i j}\right)^{*}=\left(\overline{a_{i j}}\right)^{T}$-whenever (3.4) admits only the trivial solution. (The classical Moore-Penrose inverse is thereby obtained by putting $\mathcal{K}=\mathbb{C}$, the field of complex numbers, endowed with the usual complex conjugation $z \mapsto \bar{z}$.) On the other hand, it is easy to see that the condition that (3.4) has only the trivial solution is necessary: if $\left(a_{1}, \ldots, a_{n}\right)$ were a non-trivial solution to (3.4), then the matrix formed by $n$ identical rows $\left(a_{1}, \ldots, a_{n}\right)$ would have no Moore-Penrose inverse.

The proof of the main result of this subsection requires an explicit calculation of the Moore-Penrose inverses of certain rank 1 matrices. Thus, we present a simple method for such a calculation. For a row vector $a=\left(a_{1}, \ldots, a_{n}\right) \in K^{n}$, where $\mathcal{K}=\langle K,+, \cdot\rangle$ is a field with an involution $x \mapsto \bar{x}$, let $a^{*}$ denote the column vector $\left(\overline{a_{1}}, \ldots, \overline{a_{n}}\right)^{T}$. It is easy to see that any $n \times n$-matrix $A$ of rank 1 over $\mathcal{K}$ can be represented as $A=b^{*} c$ for some non-zero row vectors $b, c \in K^{n}$. Provided that (3.4) admits only the trivial solution in $K^{n}$, one gets $A^{\dagger}$ as follows:

$$
A^{\dagger}=c^{*}\left(c c^{*}\right)^{-1}\left(b b^{*}\right)^{-1} b .
$$

Here $b b^{*}$ and $c c^{*}$ are non-zero elements of $\mathcal{K}$, so their inverses in $\mathcal{K}$ exist. In order to justify (3.5), it suffices to check that the right hand side of (3.5) satisfies the simultaneous equations (3.1) with the matrix $A$ in the role of $a$, and this is straightforward. Note that formula (3.5) immediately shows that $A^{\dagger}$ is a scalar multiple of $A^{*}=c^{*} b$, namely

$$
A^{\dagger}=\frac{1}{c c^{*} \cdot b b^{*}} A^{*} .
$$

So, we can formulate one of the highlights of the section-a result that reveals an unexpected feature of a rather classical and well studied object.

Theorem 3.3. Let $\mathcal{K}=\langle K,+, \cdot\rangle$ be a field having an involution $x \mapsto \bar{x}$ for which the equation $x \bar{x}+y \bar{y}=0$ has only the trivial solution $(x, y)=(0,0)$ in $K^{2}$. Then the unary semigroup $\left\langle\mathrm{M}_{2}(\mathcal{K}), \cdot,{ }^{\dagger}\right\rangle$ of all $2 \times 2$-matrices over $\mathcal{K}$ endowed with MoorePenrose inversion ${ }^{\dagger}$-subject to the involution $\left(a_{i j}\right) \mapsto\left(a_{i j}\right)^{*}=\left(\bar{a}_{i j}\right)^{T}$-has no finite basis of identities.

Proof. Set $\mathcal{S}=\left\langle\mathrm{M}_{2}(\mathcal{K}), \cdot,{ }^{\dagger}\right\rangle$. By Theorem 2.2 and Lemma 2.1 it is sufficient to show that

1) $\mathcal{K}_{3} \in \operatorname{var} \mathcal{S}$,

2) there exists a group $\mathcal{G} \in \operatorname{var} \mathcal{S}$ such that $\mathcal{G} \notin \operatorname{var} \mathrm{H}(\mathcal{S})$. 
In order to prove 1$)$, consider the following sets of rank 1 matrices in $\mathrm{M}_{2}(\mathcal{K})$ :

$$
\begin{aligned}
& H_{11}=\left\{\left(\begin{array}{ll}
x & x \\
x & x
\end{array}\right)\right\}, \quad H_{12}=\left\{\left(\begin{array}{ll}
x & 0 \\
x & 0
\end{array}\right)\right\}, \quad H_{13}=\left\{\left(\begin{array}{ll}
0 & x \\
0 & x
\end{array}\right)\right\}, \\
& H_{21}=\left\{\left(\begin{array}{ll}
x & x \\
0 & 0
\end{array}\right)\right\}, \quad H_{22}=\left\{\left(\begin{array}{ll}
x & 0 \\
0 & 0
\end{array}\right)\right\}, \quad H_{23}=\left\{\left(\begin{array}{ll}
0 & x \\
0 & 0
\end{array}\right)\right\} \text {, } \\
& H_{31}=\left\{\left(\begin{array}{ll}
0 & 0 \\
x & x
\end{array}\right)\right\}, \quad H_{32}=\left\{\left(\begin{array}{ll}
0 & 0 \\
x & 0
\end{array}\right)\right\}, \quad H_{33}=\left\{\left(\begin{array}{ll}
0 & 0 \\
0 & x
\end{array}\right)\right\},
\end{aligned}
$$

where in each case $x$ runs over $K \backslash\{0\}$. Observe that $\mathcal{K}$ cannot be of characteristic 2, since the equation $x \bar{x}+y \bar{y}=0$ has only the trivial solution in $K^{2}$. Taking this into account, a straightforward calculation shows that

$$
H_{i j} \cdot H_{k \ell}= \begin{cases}H_{i \ell} & \text { if }(j, k) \neq(2,3),(3,2), \\ 0 & \text { otherwise. }\end{cases}
$$

Hence the set

$$
T=\bigcup_{1 \leq i, j \leq 3} H_{i j} \cup\{0\}
$$

is closed under multiplication, so forms a subsemigroup $\mathcal{T}$ of $\mathcal{S}$, and the partition $\mathscr{H}$ of $T$ into the classes $H_{i j}$ and $\{0\}$ is a congruence on $\mathcal{T}$. Equation (3.6) shows that

$$
H_{i j}^{\dagger}=H_{j i}
$$

We see that $T$ is closed under Moore-Penrose inversion and $\mathscr{H}$ respects ${ }^{\dagger}$, thus is a congruence on the unary semigroup $\mathcal{T}^{\prime}=\left\langle T, \cdot,{ }^{\dagger}\right\rangle$. Now comparing (3.8) and (3.9) with the multiplication and inversion rules in $\mathcal{K}_{3}$ (see (1.1)), we conclude that $\mathcal{T}^{\prime} / \mathscr{H}$ and $\mathcal{K}_{3}$ are isomorphic as unary semigroups. Hence $\mathcal{K}_{3}$ is in var $\mathcal{S}$.

For 2) we merely let $\mathrm{GL}_{2}(\mathcal{K})$, the group of all invertible $2 \times 2$-matrices over $\mathcal{K}$, play the role of $\mathcal{G}$. Since Moore-Penrose inversion on $\mathrm{GL}_{2}(\mathcal{K})$ coincides with usual matrix inversion, we observe that $\mathrm{GL}_{2}(\mathcal{K})$ is a unary subsemigroup of $\mathcal{S}$. Moreover, since $A A^{\dagger}$ is the identity matrix for every invertible matrix $A$, we conclude that, with the exception of the identity matrix, the Hermitian subsemigroup $\mathrm{H}(\mathcal{S})$ contains only matrices of rank at most 1 , that is, $\mathrm{H}(\mathcal{S}) \subseteq \mathrm{L}_{2}^{1}(\mathcal{K})$, the set of all matrices of rank at most 1 with the identity matrix adjoined. By Corollary 1.2 the semigroup $\left\langle\mathrm{L}_{2}^{1}(\mathcal{K}), \cdot\right\rangle$ satisfies the identity $x^{2} y x=x y x^{2}$. Consequently, each group in var $\mathrm{H}(\mathcal{S})$ is abelian, while the group $\mathrm{GL}_{2}(\mathcal{K})$ is non-abelian. Thus, $\mathrm{GL}_{2}(\mathcal{K})$ is contained in $\operatorname{var} \mathcal{S}$ but not in $\operatorname{var} \mathrm{H}(\mathcal{S})$, as required.

Remark 3.1. Apart from any subfield of $\mathbb{C}$ closed under complex conjugation, Theorem 3.3 applies, for instance, to finite fields $\mathcal{K}=\langle K,+, \cdot\rangle$ for which $|K| \equiv 3(\bmod 4)$, endowed with the trivial involution $x \mapsto \bar{x}=x$; the latter follows from the fact that the equation $x^{2}+1=0$ admits no solution in $\mathcal{K}$ if and only if $|K| \equiv 3(\bmod 4)$ (cf. [32, Theorem 3.75]). Moreover, by slightly changing the arguments one can show an analogous result for $\mathcal{K}$ being any skew-field of quaternions closed under conjugation. 
The reader may ask whether or not the restriction on the size of matrices is essential in Theorem 3.3. For some fields, it definitely is. For instance, for finite fields with the trivial involution $x \mapsto \bar{x}=x$, no extension of Theorem 3.3 to $n \times n$-matrices with $n>2$ is possible simply because the Moore-Penrose inverse is only a partial operation in this case. Indeed, it is a well known corollary of the Chevalley-Warning theorem (see, e.g., [52, Corollary 2 in $\S 1.2]$ ) that the equation $x_{1}^{2}+\cdots+x_{n}^{2}=0$ (that is (3.4) with the trivial involution) admits a non-trivial solution in any finite field whenever $n>2$.

The situation is somewhat more complicated for subfields of $\mathbb{C}$. Theorem 2.2 does not apply here because of the following obstacle. It is well known (see, for example, $[34$, p. 101]) that the two matrices

$$
\zeta=\left(\begin{array}{ll}
1 & 0 \\
2 & 1
\end{array}\right) \quad \text { and } \quad \eta=\left(\begin{array}{ll}
1 & 2 \\
0 & 1
\end{array}\right)
$$

generate a free subgroup of $\left\langle\mathrm{SL}_{2}(\mathbb{Z}), \cdot,^{-1}\right\rangle$. On the other hand, it is easy to verify that for any subfield $\mathcal{K}$ of $\mathbb{C}$ closed under complex conjugation, the mapping $\left\langle\mathrm{SL}_{2}(\mathbb{Z}), \cdot,{ }^{-1}\right\rangle \rightarrow$

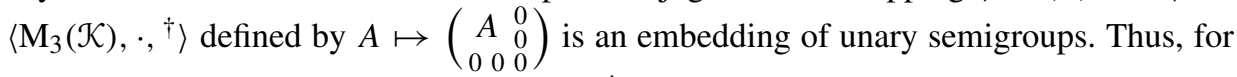
$n>2$, the unary semigroup $\left\langle\operatorname{Sing}_{n}(\mathcal{K}), \cdot,{ }^{\dagger}\right\rangle$ of singular $n \times n$-matrices contains a free non-abelian group, whence every group belongs to the unary semigroup variety generated by $\left\langle\operatorname{Sing}_{n}(\mathcal{K}), \cdot,{ }^{\dagger}\right\rangle$. Now we observe that $\operatorname{Sing}_{n}(\mathcal{K})$ is contained in the Hermitian subsemigroup of $\left\langle\mathrm{M}_{n}(\mathcal{K}), \cdot,{ }^{\dagger}\right\rangle$. Indeed, it was proved in [16] (see also [2] for a recent elementary proof) that the semigroup $\left\langle\operatorname{Sing}_{n}(\mathcal{K}), \cdot\right\rangle$ is generated by idempotent matrices. For an arbitrary idempotent matrix $A \in \mathrm{M}_{n}(\mathcal{K})$, let

$$
N(A)=\left\{x \in K^{n} \mid x A=0\right\} \quad \text { and } \quad F(A)=\left\{x \in K^{n} \mid x A=x\right\}
$$

be the null-space and the fixed-point-space of $A$, respectively. Now consider two matrices of orthogonal projectors: $P_{1}$, the matrix of the orthogonal projector onto $F(A)$, and $P_{2}$, the matrix of the orthogonal projector onto $N(A)^{\perp}$. As any orthogonal projector matrix $P$ satisfies $P=P^{2}=P^{\dagger}$, both $P_{1}=P_{1} P_{1}^{\dagger}$ and $P_{2}=P_{2} P_{2}^{\dagger}$ belong to the Hermitian subsemigroup $\mathrm{H}\left(\mathrm{M}_{n}(\mathcal{K})\right.$ ), but then $A$ also belongs to $\mathrm{H}\left(\mathrm{M}_{n}(\mathcal{K})\right)$ since $A=\left(P_{1} P_{2}\right)^{\dagger}$ (see [37, Exercise 5.15.9a]). Thus, $\operatorname{Sing}_{n}(\mathcal{K}) \subseteq \mathrm{H}\left(\mathrm{M}_{n}(\mathcal{K})\right)$, whence no group $\mathcal{G}$ can satisfy the condition of Theorem 2.2.

However, the fact that Theorem 2.2 cannot be applied to, say, the unary semigroup $\left\langle\mathrm{M}_{3}(\mathbb{C}), \cdot{ }^{\dagger}\right\rangle$ does not yet mean that the identities of this semigroup are finitely based. We thus have the following open question.

Problem 3.1. Is the unary semigroup $\left\langle\mathrm{M}_{n}(\mathcal{K}), \cdot{ }^{\dagger}\right\rangle$ not finitely based for each subfield $\mathcal{K}$ of $\mathbb{C}$ closed under complex conjugation and for all $n>2$ ?

It is known that $\left\langle\mathrm{M}_{n}(\mathcal{K}), \cdot,{ }^{\dagger}\right\rangle$ satisfies rather involved identities (see [7] for an example), so the conjecture that these identities admit no finite basis looks quite natural. In connection with Problem 3.1, we also observe that the proofs of Theorem 3.3 and Corollary 1.2 readily yield the following: 
Remark 3.2. For each conjugation-closed subfield $\mathcal{K}$ of $\mathbb{C}$ and for all $n>2$, the unary semigroup $\left\langle\mathrm{L}_{n}(\mathcal{K}) \cup G, \cdot,{ }^{\dagger}\right\rangle$ consisting of all matrices of rank at most 1 and all matrices from some non-abelian subgroup $\left\langle G, \cdot,{ }^{-1}\right\rangle$ of $\left\langle\mathrm{GL}_{n}(\mathcal{K}), \cdot,{ }^{-1}\right\rangle$ has no finite identity basis.

Another natural related structure is the semigroup $\mathrm{M}_{n}(\mathcal{K})$ endowed with both unary operations ${ }^{\dagger}$ and ${ }^{*}$. Recall that the Moore-Penrose inverse is in fact defined in terms of identities involving both these operations-namely, Proposition 3.2 implies that the algebraic structure $\left\langle\mathrm{M}_{n}(\mathcal{K}), \cdot,{ }^{\dagger},{ }^{*}\right\rangle$ satisfies the identities

$$
x x^{\dagger} x=x, \quad x^{\dagger} x x^{\dagger}=x^{\dagger}, \quad\left(x x^{\dagger}\right)^{*}=x x^{\dagger}, \quad\left(x^{\dagger} x\right)^{*}=x^{\dagger} x
$$

and these identities uniquely determine the operation $A \mapsto A^{\dagger}$. This might incline one to conjecture that the identities (3.11) together with the identities $(x y)^{*}=y^{*} x^{*}$ and $\left(x^{*}\right)^{*}=x$ form a basis for $\operatorname{Eq}\left\langle\mathrm{M}_{n}(\mathcal{K}), \cdot,{ }^{\dagger},{ }^{*}\right\rangle$. However our techniques show that this is not the case at least for $n=2$.

Theorem 3.4. Let $\mathcal{K}$ be a field as in Theorem 3.3 ; then $\left\langle\mathrm{M}_{2}(\mathcal{K}), \cdot,{ }^{\dagger},{ }^{*}\right\rangle$ is not finitely based as an algebraic structure of type $(2,1,1)$.

Proof. The characteristic of $\mathcal{K}$ is not 2, whence the group

$$
\mathcal{G}=\left\{A \in \mathrm{GL}_{2}(\mathcal{K}) \mid A^{\dagger}=A^{*}\right\}
$$

is non-abelian. Indeed, on the prime subfield of $\mathcal{K}$, the involution $x \mapsto \bar{x}$ is the identity automorphism; so, for matrices over the prime subfield, conjugation ${ }^{*}$ coincides with transposition, and thus, for example, $\left(\begin{array}{ll}0 & 1 \\ 1 & 0\end{array}\right)$ and $\left(\begin{array}{cc}0 & -1 \\ 1 & 0\end{array}\right)$ are two non-commuting members of $\mathcal{G}$. Set $\mathcal{A}=\left\langle\mathrm{M}_{2}(\mathcal{K}), \cdot,{ }^{\dagger},{ }^{*}\right\rangle$; the algebraic structure $\left\langle G, \cdot,{ }^{\dagger},{ }^{*}\right\rangle$, that is, the group $\mathcal{G}$ with inversion taken twice as unary operation, belongs to var $\mathcal{A}$. Now, as in the proof of Theorem 3.3, consider the set

$$
T=\bigcup_{1 \leq i, j \leq 3} H_{i j} \cup\{0\}
$$

where $H_{i j}$ are defined via (3.7). Obviously, $H_{i j}^{*}=H_{j i}$ whence $\mathcal{T}=\left\langle T, \cdot,{ }^{\dagger},{ }^{*}\right\rangle$ is a substructure of $\mathcal{A}$ and the partition $\mathscr{H}$ of $T$ into the classes $H_{i j}$ and $\{0\}$ is a congruence on this substructure. The quotient $\mathcal{T} / \mathscr{H}$ is then isomorphic to the semigroup $\mathcal{K}_{3}$ endowed twice with its unary operation. We conclude that $\mathcal{K}_{3}$ treated this way also belongs to $\operatorname{var} \mathcal{A}$. By Corollary 1.2 the identity $x^{2} y x=x y x^{2}$ holds in $\mathrm{H}(\mathcal{A})$ (by which we mean the substructure of $\mathcal{A}$ generated by all elements of the form $A A^{\dagger}$ ). Now construct the semigroups $\mathcal{T}_{k}$ (by use of the identity $x^{2} y x=x y x^{2}$ ) as in step 2 in the proof of Theorem 2.2 and endow each of them twice with its unary operation. The arguments in steps 3 and 4 in the proof then show that $\mathcal{T}_{k}$ does not belong to var $\mathcal{A}$ while each $k$-generated substructure of $\mathcal{T}_{k}$ does belong to var $\mathcal{A}$. Thus, $\mathcal{T}_{k}$ can play the role of critical structures for $\operatorname{var} \mathcal{A}$ and the desired conclusion follows by reasoning as in step 1 in the proof of Theorem 2.2.

Also in this setting, our result gives rise to a natural question.

Problem 3.2. Is the algebraic structure $\left\langle\mathrm{M}_{n}(\mathcal{K}), \cdot,{ }^{\dagger},{ }^{*}\right\rangle$ of type $(2,1,1)$ not finitely based for each subfield $\mathcal{K}$ of $\mathbb{C}$ closed under complex conjugation and for all $n>2$ ? 
Here an observation similar to Remark 3.2 can be stated: for each conjugation-closed subfield $\mathcal{K} \subseteq \mathbb{C}$ and for all $n>2$, the algebraic structure $\left\langle\mathrm{L}_{n}(\mathcal{K}) \cup \mathrm{GL}_{n}(\mathcal{K}), \cdot,{ }^{\dagger},{ }^{*}\right\rangle$ consisting of all matrices of rank at most 1 and all invertible matrices has no finite identity basis.

\subsection{Matrix semigroups with transposition over infinite fields}

Here we show that the involutory semigroup $\left\langle\mathrm{M}_{n}(\mathcal{K}), \cdot,{ }^{T}\right\rangle$ is finitely based for any infinite field $\mathcal{K}$. More precisely, we verify that all identities holding in $\left\langle\mathrm{M}_{n}(\mathcal{K}), \cdot,{ }^{T}\right\rangle$ follow from the associativity and the involution laws $(x y)^{T}=y^{T} x^{T},\left(x^{T}\right)^{T}=x$. This is an involutory analogue of a result from [22] mentioned in the introduction; the proof given there does not immediately show the intended analogue, but the ideas below are inspired by the arguments in [22].

Let us start with an auxiliary construction and consider first, for an arbitrary field $\mathcal{K}$, the set $\mathrm{M}_{2}(\mathcal{K}[x])$ of all $2 \times 2$-matrices over the polynomial ring $\mathcal{K}[x]$. A matrix

$$
\left(\begin{array}{ll}
p_{11} & p_{12} \\
p_{21} & p_{22}
\end{array}\right)
$$

with $p_{i j}$ non-zero members of $\mathcal{K}[x]$ is said to be ascending if

$$
\operatorname{deg}\left(p_{11}\right)<\min \left\{\operatorname{deg}\left(p_{12}\right), \operatorname{deg}\left(p_{21}\right)\right\}, \quad \max \left\{\operatorname{deg}\left(p_{12}\right), \operatorname{deg}\left(p_{21}\right)\right\}<\operatorname{deg}\left(p_{22}\right),
$$

and descending if

$$
\operatorname{deg}\left(p_{11}\right)>\max \left\{\operatorname{deg}\left(p_{12}\right), \operatorname{deg}\left(p_{21}\right)\right\}, \quad \min \left\{\operatorname{deg}\left(p_{12}\right), \operatorname{deg}\left(p_{21}\right)\right\}>\operatorname{deg}\left(p_{22} .\right)
$$

Let Asc and Desc stand for the sets of all ascending and all descending matrices, respectively. It is straightforward to see that Asc and Desc are disjoint and both are closed under multiplication and transposition. Set

$$
A=\left(\begin{array}{cc}
1 & 0 \\
x^{2} & x
\end{array}\right)
$$

by induction one obtains

$$
A^{n}=\left(\begin{array}{cc}
1 & 0 \\
p_{n+1} & p_{n}
\end{array}\right)
$$

for polynomials $p_{n}$ and $p_{n+1}$ with $\operatorname{deg}\left(p_{n+1}\right)=n+1$ and $\operatorname{deg}\left(p_{n}\right)=n$. Further, set $B=A^{T}$; then for any $n, m \geq 1$ one gets

$$
A^{n} B^{m}=\left(\begin{array}{cc}
1 & s_{12} \\
s_{21} & s_{22}
\end{array}\right)
$$

where $\operatorname{deg}\left(s_{12}\right)=m+1, \operatorname{deg}\left(s_{21}\right)=n+1$ and $\operatorname{deg}\left(s_{22}\right)=m+n+2$, while

$$
B^{m} A^{n}=\left(\begin{array}{ll}
r_{11} & r_{12} \\
r_{21} & r_{22}
\end{array}\right)
$$

where $\operatorname{deg}\left(r_{11}\right)=m+n+2, \operatorname{deg}\left(r_{12}\right)=\operatorname{deg}\left(r_{21}\right)=m+n+1$ and $\operatorname{deg}\left(r_{22}\right)=m+n$. In particular, $A^{n} B^{m} \in$ Asc while $B^{m} A^{n} \in$ Desc. 
Lemma 3.5. For all $m, n \geq 1$, the sets Asc $\cdot A^{n}$ and Desc $\cdot B^{m}$ are disjoint.

Proof. Let $a, b, c, d, p, q$ be non-zero polynomials with

$\operatorname{deg}(a)<\min \{\operatorname{deg}(b), \operatorname{deg}(c)\}, \max \{\operatorname{deg}(b), \operatorname{deg}(c)\}<\operatorname{deg}(d)$ and $\operatorname{deg}(p)<\operatorname{deg}(q)$.

Then $\left(\begin{array}{ll}a & b \\ c & d\end{array}\right)$ is a typical matrix in Asc, and $\left(\begin{array}{ll}d & b \\ c & a\end{array}\right)$ is one in Desc; $A^{n}$ is of the form $\left(\begin{array}{ll}1 & 0 \\ q & p\end{array}\right)$ and $B^{m}$ is of the form $\left(\begin{array}{ll}1 & q \\ 0 & p\end{array}\right)$. Now

$$
\left(\begin{array}{ll}
a & b \\
c & d
\end{array}\right)\left(\begin{array}{ll}
1 & 0 \\
q & p
\end{array}\right)=\left(\begin{array}{ll}
a+b q & b p \\
c+d q & d p
\end{array}\right)
$$

Since $\operatorname{deg}(a)<\operatorname{deg}(b)$ and $\operatorname{deg}(p)<\operatorname{deg}(q)$ we obtain

$$
\operatorname{deg}(a+b q)=\operatorname{deg}(b q)>\operatorname{deg}(b p) .
$$

In particular, for any $C=\left(c_{i j}\right) \in \mathrm{Asc} \cdot A^{n}$ we get

$$
\operatorname{deg}\left(c_{11}\right)>\operatorname{deg}\left(c_{12}\right) .
$$

On the other hand,

$$
\left(\begin{array}{ll}
d & b \\
c & a
\end{array}\right)\left(\begin{array}{ll}
1 & q \\
0 & p
\end{array}\right)=\left(\begin{array}{ll}
d & d q+b p \\
c & c q+a p
\end{array}\right)
$$

Here we have

$$
\operatorname{deg}(d)<\operatorname{deg}(d q)=\operatorname{deg}(d q+b p) .
$$

Again, this shows that for any $D=\left(d_{i j}\right) \in \operatorname{Desc} \cdot B^{m}$,

$$
\operatorname{deg}\left(d_{11}\right)<\operatorname{deg}\left(d_{12}\right) .
$$

Conditions (3.12) and (3.13) immediately imply that Asc $\cdot A^{n} \cap$ Desc $\cdot B^{m}=\emptyset$.

We are able to draw our first conclusion.

Proposition 3.6. Let $u=u(a, b)$ and $v=v(a, b) \in\{a, b\}^{+}$be two distinct words, $A=\left(\begin{array}{cc}1 & 0 \\ x^{2} & x\end{array}\right) \in \mathrm{M}_{2}(\mathcal{K}[x])$ and $B=A^{T}$. Then $u(A, B) \neq v(A, B)$.

Proof. Suppose that $u(A, B)=v(A, B)$. The matrices $A$ and $B$ are invertible as matrices over the field of rational functions over $\mathcal{K}$, so we can cancel the longest common prefix and the longest common suffix of the words $u$ and $v$, obtaining new words $u^{\prime}$ and $v^{\prime}$ that still satisfy $u^{\prime}(A, B)=v^{\prime}(A, B)$. Observe that neither $u^{\prime}$ nor $v^{\prime}$ is empty because otherwise $A$ and/or $B$ would be invertible over the polynomial ring $\mathcal{K}[x]$, which is not true. Thus, we may assume that $u$ and $v$ start and end with different symbols, and this means that the words are of either of the following two forms:

$$
\begin{aligned}
& u=a^{n_{1}} b^{m_{1}} \cdots a^{n_{s}} b^{m_{s}} \quad \text { and } \quad v=b^{\ell_{1}} a^{k_{1}} \cdots b^{\ell_{t}} a^{k_{t}}, \\
& u=a^{n_{1}} b^{m_{1}} \cdots a^{n_{s-1}} b^{m_{s-1}} a^{n_{s}} \quad \text { and } \quad v=b^{\ell_{1}} a^{k_{1}} \cdots b^{\ell_{t-1}} a^{k_{t-1}} b^{\ell_{t}} \text {, }
\end{aligned}
$$

with $s, t \geq 1$ and all $n_{i}, m_{i}, k_{i}, \ell_{i} \geq 1$. In case (3.14), $u(A, B) \in$ Asc and $v(A, B) \in$ Desc, while in case (3.15), $u(A, B) \in$ Asc $\cdot A^{n_{s}}$ and $v(A, B) \in$ Desc $\cdot B^{k_{t}}$. In any case, using Lemma 3.5 we obtain $u(A, B) \neq v(A, B)$, a contradiction. 
Theorem 3.7. For each infinite field $\mathcal{K}$, all the identities of the involutory semigroup $\left\langle\mathrm{M}_{n}(\mathcal{K}), \cdot{ }^{T}\right\rangle$ follow from the laws $(x y) z=x(y z),(x y)^{T}=y^{T} x^{T},\left(x^{T}\right)^{T}=x$.

Proof. If we call identities that follow from the associativity and the involution laws trivial, our claim amounts to saying that $\left\langle\mathrm{M}_{n}(\mathcal{K}), \cdot,{ }^{T}\right\rangle$ does not satisfy any non-trivial involutory identity. We show that this already holds for the involutory semigroup $\mathcal{G} \mathcal{L}_{2}(\mathcal{K})=$ $\left\langle\mathrm{GL}_{2}(\mathcal{K}), \cdot,{ }^{T}\right\rangle$, which obviously embeds into $\left\langle\mathrm{M}_{n}(\mathcal{K}), \cdot,{ }^{T}\right\rangle$ for each $n$.

It is known (and easy to verify) that the free involutory semigroup with one generator, say $z$, contains as a unary subsemigroup a free involutory semigroup with countably many generators, namely $\mathcal{F J}(Z)$ where

$$
Z=\left\{z z^{*} z, z\left(z^{*}\right)^{2} z, \ldots, z\left(z^{*}\right)^{n} z, \ldots\right\} .
$$

Therefore we only need to verify that $\mathcal{G} \mathcal{L}_{2}(\mathcal{K})$ satisfies no non-trivial involutory identity in one letter $z$. Such an identity can be written as $u\left(z, z^{T}\right)=v\left(z, z^{T}\right)$ with $u$ and $v$ being two distinct words. Proposition 3.6 implies that $u(A, B) \neq v(A, B)$ in $\mathrm{M}_{2}(\mathcal{K}[x])$. Setting

$$
u(A, B)=\left(\begin{array}{ll}
u_{11} & u_{12} \\
u_{21} & u_{22}
\end{array}\right) \quad \text { and } \quad v(A, B)=\left(\begin{array}{ll}
v_{11} & v_{12} \\
v_{21} & v_{22}
\end{array}\right)
$$

we see that for some indices $i$ and $j$ the polynomials $u_{i j}$ and $v_{i j}$ are distinct, so $u_{i j}-v_{i j}$ is a non-zero polynomial. Now take any $\lambda \in \mathcal{K}$ and set $z(\lambda)=\left(\begin{array}{cc}1 & 0 \\ \lambda^{2} & \lambda\end{array}\right)$. If

$$
u\left(z(\lambda), z(\lambda)^{T}\right)=v\left(z(\lambda), z(\lambda)^{T}\right)
$$

then $\lambda$ must be a root of the non-zero polynomial $u_{i j}-v_{i j}$, so (3.16) can hold only for finitely many $\lambda$ in $\mathcal{K}$. Since $\mathcal{K}$ is infinite, equality (3.16) fails for all but finitely many $\lambda$, and so, in particular, the identity $u\left(z, z^{T}\right)=v\left(z, z^{T}\right)$ fails in $\mathcal{G} \mathcal{L}_{2}(\mathcal{K})$.

Similarly, it can be shown that the involutory semigroup $\left\langle\mathrm{M}_{n}(R), \cdot,{ }^{*}\right\rangle$ is finitely based for each subring $R \subseteq \mathbb{C}$ closed under complex conjugation-here ${ }^{*}$ stands for the complexconjugate transposition $\left(a_{i j}\right)^{*}=\left(\overline{a_{i j}}\right)^{T}$. Indeed, we have already mentioned that the two matrices $\zeta$ and $\eta$ in (3.10) generate a free subgroup of $\left\langle\operatorname{SL}_{2}(\mathbb{Z}), \cdot,{ }^{-1}\right\rangle$ and hence a free subsemigroup of $\left\langle\mathrm{SL}_{2}(\mathbb{Z}), \cdot\right\rangle$. But $\eta=\zeta^{*}$, so the involutory subsemigroup in $\left\langle\mathrm{SL}_{2}(\mathbb{Z}), \cdot,{ }^{*}\right\rangle$ generated by $\zeta$ is isomorphic to the free involutory semigroup $\mathcal{F J}(\{\zeta\})$.

We note that Theorem 2.2 and Corollary 1.2 prove the non-existence of a finite identity bases for the unary subsemigroup of $\left\langle\mathrm{M}_{n}(\mathbb{C}), \cdot{ }^{*}\right\rangle\left[\operatorname{respectively}\left\langle\mathrm{M}_{n}(\mathbb{R}), \cdot,{ }^{T}\right\rangle\right]$ that consists of all matrices of rank at most 1 together with all unitary [respectively all orthogonal] matrices.

\subsection{Matrix semigroups with transposition over finite fields}

Now we demonstrate that the case of finite fields is completely opposite to the one of infinite fields with respect to the finite basis problem for matrix semigroups with transposition. We start by considering $2 \times 2$-matrices. Here Theorem 2.2 solves the finite basis problem in the negative for the involutory semigroup $\left\langle\mathrm{M}_{2}(\mathcal{K}), \cdot,{ }^{T}\right\rangle$ for each finite field $\mathcal{K}$ 
except $\mathcal{K}=\mathbb{F}_{2}$, the 2-element field. (The exception is due to the fact that the involutory semigroup $\left\langle\mathrm{M}_{2}\left(\mathbb{F}_{2}\right), \cdot,{ }^{T}\right\rangle$ satisfies the identity

$$
\left(x x^{T}\right)^{3}\left(y y^{T}\right)^{3}=\left(y y^{T}\right)^{3}\left(x x^{T}\right)^{3},
$$

which does not hold in $\mathcal{K}_{3}$; consequently, $\mathcal{K}_{3}$ is not in $\operatorname{var}\left\langle\mathrm{M}_{2}\left(\mathbb{F}_{2}\right), \cdot,{ }^{T}\right\rangle$ and Theorem 2.2 does not apply here.) In the following theorem, we shall demonstrate the application of Theorem 2.2 only in the case when $\mathcal{K}$ has odd characteristic. With some additional effort we could also include the case when the characteristic of $\mathcal{K}$ is 2 and $|K| \geq 4$. We shall omit this since that case will be covered by a different kind of proof later.

Theorem 3.8. For each finite field $\mathcal{K}$ of odd characteristic, the involutory semigroup $\left\langle\mathrm{M}_{2}(\mathcal{K}), \cdot,{ }^{T}\right\rangle$ has no finite identity basis.

Proof. Let $\mathcal{S}=\left\langle\mathrm{M}_{2}(\mathcal{K}), \cdot{ }^{T}\right\rangle$. As in the proof of Theorem 3.3 one shows that $\mathcal{K}_{3}$ is in var $\mathcal{S}$. Furthermore, let $d$ be the exponent of the group $\mathrm{GL}_{2}(\mathcal{K})$. By Corollary 1.2, each group in $\mathrm{P}_{d}(\operatorname{var} \mathcal{S})=\operatorname{var} \mathrm{P}_{d}(\mathcal{S})$ satisfies the identity $x^{2} y x=x y x^{2}$ and therefore is abelian. On the other hand, as in the proof of Theorem 3.4, the group $\mathcal{G}=\left\{A \in \mathrm{GL}_{2}(\mathcal{K}) \mid\right.$ $\left.A^{T}=A^{-1}\right\}$ is in var $\mathcal{S}$ but is non-abelian. Thus, Theorem 2.2 applies.

The next theorem contains the even characteristic case and yields, in fact, a stronger assertion.

Theorem 3.9. For each finite field $\mathcal{K}=\langle K,+, \cdot\rangle$ with $|K| \not \equiv 3(\bmod 4)$, the involutory semigroup $\left\langle\mathrm{M}_{2}(\mathcal{K}), \cdot,{ }^{T}\right\rangle$ is inherently non-finitely based.

Proof. As mentioned in Remark 3.1, there exists $x \in K$ for which $1+x^{2}=0$. Now consider the following matrices:

$$
\begin{array}{ccc}
H_{11}=\left(\begin{array}{cc}
1 & x \\
x & x^{2}
\end{array}\right), & H_{12}=\left(\begin{array}{ll}
1 & 0 \\
x & 0
\end{array}\right), & H_{21}=\left(\begin{array}{ll}
1 & x \\
0 & 0
\end{array}\right), \quad H_{22}=\left(\begin{array}{ll}
1 & 0 \\
0 & 0
\end{array}\right), \\
I=\left(\begin{array}{ll}
1 & 0 \\
0 & 1
\end{array}\right), & O=\left(\begin{array}{ll}
0 & 0 \\
0 & 0
\end{array}\right) .
\end{array}
$$

Then the set $M=\left\{H_{11}, H_{12}, H_{21}, H_{22}, I, O\right\}$ is closed under multiplication and transposition, hence $\mathcal{M}=\left\langle M, \cdot,{ }^{T}\right\rangle$ is an involutory subsemigroup of $\left\langle\mathrm{M}_{2}(\mathcal{K}), \cdot,{ }^{T}\right\rangle$. The mapping $\mathcal{T} \mathcal{A}_{2}^{1} \rightarrow \mathcal{M}$ given by

$$
(i, j) \mapsto H_{i j}, \quad 0 \mapsto O, \quad 1 \mapsto I
$$

is an isomorphism of involutory semigroups. The result now follows from Corollary 2.8.

The case of matrix semigroups of size greater than 2 is similar.

Theorem 3.10. For each finite field $\mathcal{K}$, the involutory semigroup $\left\langle\mathrm{M}_{n}(\mathcal{K}), \cdot,{ }^{T}\right\rangle$ with $n \geq$ 3 is inherently non-finitely based. 
Proof. It follows from the Chevalley-Warning theorem [52, Corollary 2 in $\S 1.2$ ] that there exist $x, y \in K$ satisfying $1+x^{2}+y^{2}=0$. Now consider the following matrices:

$$
\begin{array}{ccc}
H_{11}=\left(\begin{array}{ccc}
1 & x & y \\
x & x^{2} & x y \\
y & x y & y^{2}
\end{array}\right), & H_{12}=\left(\begin{array}{lll}
1 & 0 & 0 \\
x & 0 & 0 \\
y & 0 & 0
\end{array}\right), & H_{21}=\left(\begin{array}{lll}
1 & x & y \\
0 & 0 & 0 \\
0 & 0 & 0
\end{array}\right), \\
H_{22}=\left(\begin{array}{lll}
1 & 0 & 0 \\
0 & 0 & 0 \\
0 & 0 & 0
\end{array}\right), & I=\left(\begin{array}{lll}
1 & 0 & 0 \\
0 & 1 & 0 \\
0 & 0 & 1
\end{array}\right), & O=\left(\begin{array}{lll}
0 & 0 & 0 \\
0 & 0 & 0 \\
0 & 0 & 0
\end{array}\right) .
\end{array}
$$

Again, the set $M=\left\{H_{11}, H_{12}, H_{21}, H_{22}, I, O\right\}$ is closed under multiplication and transposition, and as in the previous proof, $\mathcal{M}=\left\langle M, \cdot,{ }^{T}\right\rangle$ forms an involutory subsemigroup of $\left\langle\mathrm{M}_{3}(\mathcal{K}), \cdot,{ }^{T}\right\rangle$ that is isomorphic with $\mathcal{T A}_{2}^{1}$. Hence $\left\langle\mathrm{M}_{3}(\mathcal{K}), \cdot,{ }^{T}\right\rangle$ is inherently nonfinitely based. The assertion for $\mathrm{M}_{n}(\mathcal{K})$ for $n \geq 3$ now follows in an obvious way.

Remark 3.3. The statements of Theorems 3.8, 3.9, 3.10 remain valid if the unary operation $A \mapsto A^{T}$ is replaced with an operation of the form $A \mapsto A^{\sigma T}$ for any automorphism $\sigma$ of $\mathcal{K}$, where $\left(a_{i j}\right)^{\sigma T}:=\left(a_{i j}^{\sigma}\right)^{T}$.

We are ready to prove the main result of this subsection. With the exception of the 'only if' part of item 2, this is a summary of Theorems 3.8, 3.9, and 3.10.

Theorem 3.11. Let $\mathcal{K}=\langle K,+, \cdot\rangle$ be a finite field. Then

1. the involutory semigroup $\left\langle\mathrm{M}_{n}(\mathcal{K}), \cdot,{ }^{T}\right\rangle$ is not finitely based;

2. the involutory semigroup $\left\langle\mathrm{M}_{n}(\mathcal{K}), \cdot,{ }^{T}\right\rangle$ is inherently non-finitely based if and only if either $n \geq 3$ or $n=2$ and $|K| \not \equiv 3(\bmod 4)$.

Proof. The only assertion of this theorem not covered by our previous results is that $\left\langle\mathrm{M}_{2}(\mathcal{K}), \cdot{ }^{T}\right\rangle$ is not inherently non-finitely based if $|K| \equiv 3(\bmod 4)$. We employ Proposition 2.9 to prove this.

Recall that the condition $|K| \equiv 3(\bmod 4)$ corresponds precisely to the case when each matrix $A$ in $\left\langle\mathrm{M}_{2}(\mathcal{K}), \cdot{ }^{T}\right\rangle$ admits a Moore-Penrose inverse $A^{\dagger}$ (Remark 3.1). Let $A$ be a matrix of rank 1; by (3.6) there exists a scalar $\alpha \in \mathcal{K} \backslash\{0\}$ such that $\alpha A^{\dagger}=A^{T}$. Let $r=|K|-1$; then $\alpha^{r}=1$. Since the multiplicative subgroup of $\mathcal{K}$ is a cyclic subgroup of $\mathrm{GL}_{2}(\mathcal{K})$, the number $r$ divides the exponent $d$ of $\mathrm{GL}_{2}(\mathcal{K})$, so $\alpha^{d}=1$. Consequently,

$$
A\left(A^{T} A\right)^{d}=A\left(\alpha A^{\dagger} A\right)^{d}=\alpha^{d} A\left(A^{\dagger} A\right)^{d}=A .
$$

If $A \in \mathrm{GL}_{2}(\mathcal{K})$, we also have $A=A\left(A^{T} A\right)^{d}$ because $\left(A^{T} A\right)^{d}$ is the identity matrix; clearly, the equality $A=A\left(A^{T} A\right)^{d}$ also holds for the case when $A$ is the zero matrix. Summarizing, we conclude that the identity $x=x\left(x^{T} x\right)^{d}$ holds in the involutory semigroup $\left\langle\mathrm{M}_{2}(\mathcal{K}), \cdot,{ }^{T}\right\rangle$. Setting $\omega(x):=x^{T}\left(x x^{T}\right)^{d-1}$, we see that $\left\langle\mathrm{M}_{2}(\mathcal{K}), \cdot,^{T}\right\rangle$ satisfies the identity $x=x \omega(x) x$, as required by Proposition 2.9.

Remark 3.4. It is known [48, Corollary 6.2] that the matrix semigroup $\left\langle\mathbf{M}_{n}(\mathcal{K}), \cdot\right\rangle$ is inherently non-finitely based (as a plain semigroup) for every finite field $\mathcal{K}$. Thus, the involutory semigroups $\left\langle\mathrm{M}_{2}(\mathcal{K}), \cdot{ }^{T}\right\rangle$ over finite fields $\mathcal{K}$ such that $|K| \equiv 3(\bmod 4)$ provide a natural series of unary semigroups whose equational properties essentially differ from the equational properties of their semigroup reducts. 


\subsection{Matrix semigroups with symplectic transpose}

For a $2 m \times 2 m$-matrix

$$
X=\left(\begin{array}{ll}
A & B \\
C & D
\end{array}\right)
$$

with $A, B, C, D$ being $m \times m$-matrices over any field $\mathcal{K}$, the symplectic transpose $X^{S}$ is defined by

$$
X^{S}=\left(\begin{array}{cc}
D^{T} & -B^{T} \\
-C^{T} & A^{T}
\end{array}\right)
$$

(see, e.g., [44, (5.1.1)]). The symplectic transpose is an involution of $\left\langle\mathbf{M}_{2 m}(\mathcal{K}), \cdot\right\rangle$ whose properties essentially differ from those of the usual transposition. In fact, every involution of the semigroup $\left\langle\mathrm{M}_{n}(\mathcal{K}), \cdot\right\rangle$ that fixes the scalar matrices is in a certain sense similar to either the usual transposition or the symplectic transpose. ${ }^{4}$

The definition of the symplectic transpose resembles that of the involution in the twisted Brandt monoid $\mathcal{T B}_{2}^{1}$ (defined in terms of $2 \times 2$-matrices) and leads to the following application.

Theorem 3.12. The involutory semigroup $\left\langle\mathrm{M}_{2 m}(\mathcal{K}), \cdot{ }^{S}\right\rangle$ is inherently non-finitely based for each $m \geq 1$ and each finite field $\mathcal{K}=\langle K,+, \cdot\rangle$.

Proof. Consider the following sets of $2 m \times 2 m$-matrices:

$$
\begin{array}{ll}
H_{11}=\left\{ \pm\left(\begin{array}{cc}
O_{m} & I_{m} \\
O_{m} & O_{m}
\end{array}\right)\right\}, & H_{12}=\left\{ \pm\left(\begin{array}{cc}
I_{m} & O_{m} \\
O_{m} & O_{m}
\end{array}\right)\right\}, \\
H_{21}=\left\{ \pm\left(\begin{array}{cc}
O_{m} & O_{m} \\
O_{m} & I_{m}
\end{array}\right)\right\}, & H_{22}=\left\{ \pm\left(\begin{array}{cc}
O_{m} & O_{m} \\
I_{m} & O_{m}
\end{array}\right)\right\}
\end{array}
$$

where for any positive integer $k$, we denote be $I_{k}$, respectively, $O_{k}$ the identity, respectively, zero $k \times k$-matrix. Let

$$
T=\bigcup_{1 \leq i, j \leq 2} H_{i j} \cup\left\{O_{2 m}, I_{2 m}\right\} .
$$

The set $T$ is closed under multiplication and symplectic transposition, so $\left\langle T, \cdot{ }^{S}\right\rangle$ forms an involutory subsemigroup of $\left\langle\mathrm{M}_{2 m}(\mathcal{K}), \cdot,{ }^{S}\right\rangle$. On the other hand, the mapping

$$
H_{i j} \mapsto(i, j), \quad I_{2 m} \mapsto 1, \quad O_{2 m} \mapsto 0
$$

is a homomorphism of $\left\langle T, \cdot,{ }^{S}\right\rangle$ onto $\mathcal{T B}_{2}^{1}$. Altogether, the twisted Brandt monoid $\mathcal{T B}_{2}^{1}$ is a homomorphic image of an involutory subsemigroup of $\left\langle\mathrm{M}_{2 m}(\mathcal{K}), \cdot,{ }^{S}\right\rangle$.

4 We do not want to formalize this phrase in general because its meaning actually depends on some intrinsic properties of the field $\mathcal{K}$. In the simplest case, when $\mathcal{K}$ is algebraically closed of characteristic $\neq 2$, the similarity takes the strongest form: every involutory semigroup of the form $\left\langle\mathrm{M}_{n}(\mathcal{K}), \cdot,{ }^{*}\right\rangle$ such that ${ }^{*}$ fixes the scalar matrices is isomorphic to either $\left\langle\mathrm{M}_{n}(\mathcal{K}), \cdot,{ }^{T}\right\rangle$ or $\left\langle\mathrm{M}_{n}(\mathcal{K}), \cdot,{ }^{S}\right\rangle$ (in the latter case $n$ should be even). This is well known for involutions that respect the addition of matrices (see, e.g., [43, Corollary 14.2]) but it easily follows from a classical result by Khalezov [26] that every involution of the semigroup $\left\langle\mathrm{M}_{n}(\mathcal{K}), \cdot\right\rangle$ automatically preserves addition. 
Remark 3.5. It is easy to see that the involutory semigroup $\left\langle\mathrm{M}_{m}(\mathcal{K}), \cdot,{ }^{T}\right\rangle$ embeds into $\left\langle\mathrm{M}_{2 m}(\mathcal{K}), \cdot{ }^{S}\right\rangle$ via the mapping $A \mapsto\left(\begin{array}{cc}A & O_{m} \\ O_{m} & A\end{array}\right)$. Therefore, if $m>1$ and $\mathcal{K}$ is an infinite field, then $\left\langle\mathrm{M}_{2 m}(\mathcal{K}), \cdot{ }^{S}\right\rangle$ satisfies no non-trivial involutory identity and is finitely based (see Subsection 3.2). The $2 \times 2$-matrices over an infinite field satisfy non-trivial identities involving multiplication and the symplectic transpose, for instance, $x x^{S} y=y x x^{S}$ or $x x^{S}=x^{S} x$. In fact, we have verified that these two identities together with the associativity and the involution laws $(x y)^{S}=y^{S} x^{S},\left(x^{S}\right)^{S}=x$ form an identity basis for $\left\langle\mathrm{M}_{2}(\mathcal{K}), \cdot{ }^{S}\right\rangle$ with $\mathcal{K}$ infinite. (The proof of this will be published separately.) Thus, the involutory semigroup $\left\langle\mathrm{M}_{2 m}(\mathcal{K}), \cdot,{ }^{S}\right\rangle$ is finitely based if and only if $\mathcal{K}$ is an infinite field.

\subsection{Boolean matrices}

Recall that a Boolean matrix is a matrix with entries 0 and 1 only. The multiplication of such matrices is as usual, except that addition and multiplication of the entries is defined as: $a+b=\max \{a, b\}$ and $a \cdot b=\min \{a, b\}$. Let $B_{n}$ denote the set of all Boolean $n \times n$-matrices. It is well known that the semigroup $\left\langle B_{n}, \cdot\right\rangle$ is essentially the same as the semigroup of all binary relations on an $n$-element set subject to the usual composition of binary relations. The operation ${ }^{T}$ of forming the matrix transpose then corresponds to the operation of forming the dual binary relation.

Theorem 3.13. The involutory semigroup $\mathcal{B}_{n}=\left\langle B_{n}, \cdot,{ }^{T}\right\rangle$ of all Boolean $n \times n$-matrices endowed with transposition is inherently non-finitely based.

Proof. Consider the Boolean matrices

$$
\begin{aligned}
& B_{11}=\left(\begin{array}{ll}
0 & 1 \\
1 & 1
\end{array}\right), \quad B_{12}=\left(\begin{array}{ll}
1 & 0 \\
1 & 1
\end{array}\right), \quad B_{21}=\left(\begin{array}{ll}
1 & 1 \\
0 & 1
\end{array}\right), \quad B_{22}=\left(\begin{array}{ll}
1 & 1 \\
1 & 0
\end{array}\right), \\
& O=\left(\begin{array}{ll}
1 & 1 \\
1 & 1
\end{array}\right), \quad I=\left(\begin{array}{ll}
1 & 0 \\
0 & 1
\end{array}\right) \text {. }
\end{aligned}
$$

The set $M=\left\{B_{11}, B_{12}, B_{21}, B_{22}, O, I\right\}$ is closed under multiplication and transposition, so $\mathcal{M}=\left\langle M, \cdot{ }^{T}\right\rangle$ is an involutory subsemigroup of $\mathcal{B}_{2}$. The mapping $\mathcal{T B}_{2}^{1} \rightarrow \mathcal{M}$ given by

$$
(i, j) \mapsto B_{i j}, \quad 0 \mapsto O, \quad 1 \mapsto I
$$

is an isomorphism of involutory semigroups. By Corollary $2.7, \mathcal{M}$ is inherently nonfinitely based whence so is $\mathcal{B}_{2}$. Since $\mathcal{B}_{2}$ can be embedded as an involutory semigroup into $\mathcal{B}_{n}$ for each $n$, the result follows.

Remark 3.6. Our proof of Theorem 3.13 also applies to some important involutory subsemigroups of $\mathcal{B}_{n}$. In order to introduce an interesting instance, recall that Boolean $n \times n$ matrices are in a 1-1 correspondence with bipartite directed graphs whose parts are of size $n$ : the bipartite graph of a matrix $A=\left(a_{i j}\right)$ has the row set and the column set of $A$ as its parts and has an edge from the $i^{\text {th }}$ row to the $j^{\text {th }}$ column if and only if $a_{i j}=1$. If the graph of $A$ admits a perfect matching (i.e. a set of edges such that every vertex is incident to precisely one of them), then $A$ is said to be a Hall matrix (the name suggested in [51] 
is, of course, inspired by Hall's marriage theorem). It is easy to see that the collection $H B_{n}$ of all Hall $n \times n$-matrices is closed under multiplication and transposition. Since all the matrices $B_{11}, B_{12}, B_{21}, B_{22}, O, I$ from the above proof are Hall matrices, we readily conclude that the involutory semigroup $\left\langle H B_{n}, \cdot,{ }^{T}\right\rangle$ is inherently non-finitely based.

Remark 3.7. We can unify Theorem 3.13 and some results in Subsection 3.3 by considering matrices over semirings. A semiring is an algebraic structure $\mathcal{L}=\langle L,+, \cdot\rangle$ of type $(2,2)$ such that $\langle L,+\rangle$ is a commutative semigroup, $\langle L, \cdot\rangle$ is a semigroup and multiplication distributes over addition. From the proofs of Theorems 3.9 and 3.13 we see that the involutory matrix semigroup $\left\langle\mathrm{M}_{n}(\mathcal{L}), \cdot{ }^{T}\right\rangle$ over a finite semiring is inherently non-finitely based whenever the semiring $\mathcal{L}$ has a zero 0 (that is, a neutral element for $\langle L,+\rangle$ which is at the same time an absorbing element for $\langle L, \cdot\rangle)$ and satisfies one of the following two conditions:

(1) there exist (not necessarily distinct) elements $e, x \neq 0$ such that $e^{2}=e$, ex $=$ $x e=x, e+x^{2}=0$

(2) there exists an element $e \neq 0$ such that $e^{2}=e=e+e$.

We have already met an infinite series of semirings satisfying (1): it consists of the finite fields $\mathcal{K}=\langle K,+, \cdot\rangle$ with $|K| \not \equiv 3(\bmod 4)$. It should be noted that semirings satisfying (2) are even more plentiful: for example, finite distributive lattices as well as the power semirings of finite semigroups (with the subset union as addition and the subset product as multiplication) fall in this class.

A Boolean matrix $A=\left(a_{i j}\right)$ is said to be upper triangular if $a_{i j}=0$ whenever $i>j$. Let $B T_{n}$ stand for the set of all Boolean upper triangular $n \times n$-matrices. The semigroups $\left\langle B T_{n}, \cdot\right\rangle$ play an important role in the theory of formal languages (see [42]); their identities have been studied by the third author and Goldberg in [56]. Observe that this semigroup admits quite a natural unary operation: reflection with respect to the secondary diagonal (from the top right to the bottom left corner). We denote by $A^{D}$ the result of applying this operation to the matrix $A$. It is easy to see that the operation $A \mapsto A^{D}$ is in fact an involution; this follows, for instance, from the fact that $A^{D}=J A^{T} J$ where $J$ is the Boolean matrix with 1 s on the secondary diagonal and 0 s elsewhere.

Theorem 3.14. For each integer $n \geq 3$, the involutory semigroup $\mathcal{B T}_{n}=\left\langle B T_{n}, \cdot,{ }^{D}\right\rangle$ of all Boolean upper triangular $n \times n$-matrices endowed with the reflection with respect to the secondary diagonal is inherently non-finitely based.

Proof. Consider the involutory submonoid $\mathcal{M}$ in $\mathcal{B T}_{n}$ generated by the following two Boolean matrices:

$$
X=\left(\begin{array}{ccccc}
1 & 0 & \ldots & 0 & 0 \\
0 & 0 & \ldots & 0 & 0 \\
\vdots & \vdots & \ddots & \vdots & \vdots \\
0 & 0 & \ldots & 0 & 0 \\
0 & 0 & \ldots & 0 & 1
\end{array}\right) \quad \text { and } Y=\left(\begin{array}{ccccc}
1 & 1 & \ldots & 1 & 0 \\
0 & 0 & \ldots & 0 & 1 \\
\vdots & \vdots & \ddots & \vdots & \vdots \\
0 & 0 & \ldots & 0 & 1 \\
0 & 0 & \ldots & 0 & 1
\end{array}\right)
$$


Clearly, for each matrix $\left(m_{i j}\right)$ in this submonoid one has $m_{11}=m_{n n}=1$, whence the set of all matrices $\left(m_{i j}\right)$ such that $m_{1 n}=1$ forms an ideal in $\mathcal{M}$. We denote this ideal by $\mathcal{N}$. A straightforward calculation shows that, besides $X, Y$, and the identity matrix $I$, only the two matrices

$$
X Y=\left(\begin{array}{ccccc}
1 & 1 & \ldots & 1 & 0 \\
0 & 0 & \ldots & 0 & 0 \\
\vdots & \vdots & \ddots & \vdots & \vdots \\
0 & 0 & \ldots & 0 & 0 \\
0 & 0 & \ldots & 0 & 1
\end{array}\right) \quad \text { and } Y X=\left(\begin{array}{ccccc}
1 & 0 & \ldots & 0 & 0 \\
0 & 0 & \ldots & 0 & 1 \\
\vdots & \vdots & \ddots & \vdots & \vdots \\
0 & 0 & \ldots & 0 & 1 \\
0 & 0 & \ldots & 0 & 1
\end{array}\right)
$$

belong to $\mathcal{M} \backslash \mathcal{N}$. This allows one to organize the following bijection between $\mathcal{M} \backslash \mathcal{N}$ and the set of non-zero matrices in $\mathcal{T A}_{2}^{1}$ :

$$
I \mapsto\left(\begin{array}{ll}
1 & 0 \\
0 & 1
\end{array}\right), \quad X \mapsto\left(\begin{array}{ll}
1 & 0 \\
1 & 0
\end{array}\right), \quad Y \mapsto\left(\begin{array}{ll}
0 & 1 \\
0 & 0
\end{array}\right), \quad X Y \mapsto\left(\begin{array}{ll}
0 & 1 \\
0 & 1
\end{array}\right), \quad Y X \mapsto\left(\begin{array}{ll}
1 & 0 \\
0 & 0
\end{array}\right)
$$

One easily checks that extending this bijection to $\mathcal{M}$ by sending all elements from $\mathcal{N}$ to $\left(\begin{array}{ll}0 & 0 \\ 0 & 0\end{array}\right)$ yields an involutory semigroup homomorphism from $\mathcal{M}$ onto $\mathcal{T A}_{2}^{1}$. Thus, $\mathcal{T} \mathcal{A}_{2}^{1}$ as a homomorphic image of an involutory subsemigroup in $\mathcal{B T}_{n}$ belongs to the involutory semigroup variety generated by $\mathcal{B T}_{n}$. Corollary 2.8 implies that $\mathcal{B T}_{n}$ is inherently nonfinitely based.

Remark 3.8. In [56] it was shown that the semigroup $\left\langle B T_{n}, \cdot\right\rangle$ is inherently non-finitely based for $n \geq 4$. However, the construction used there does not imply the same for the involutory case. Our proof of Theorem 3.14 follows a different construction suggested for the plain semigroup case by $\mathrm{Li}$ and Luo [31]. Li and Luo have also verified that the semigroup $\left\langle B T_{2}, \cdot\right\rangle$ is finitely based. We do not know whether or not the involutory semigroup $\mathrm{BT}_{2}$ is finitely based.

Other interesting involutory semigroups of Boolean matrices include the semigroup $\mathcal{B R}_{n}=\left\langle B R_{n}, \cdot,{ }^{T}\right\rangle$ of all Boolean $n \times n$-matrices with 1 s on the main diagonal (such matrices correspond to reflexive binary relations) and the semigroup $\mathcal{B} U_{n}=\left\langle B U_{n}, \cdot{ }^{D}\right\rangle$ of all Boolean upper triangular $n \times n$-matrices with 1 s on the main diagonal. (The unary operation is the usual transpose in the former case and reflection with respect to the secondary diagonal in the latter.) Our present methods do not yet suffice to handle the finite basis problem for these unary semigroups so we just formulate

Problem 3.3. Are the involutory semigroups $\mathcal{B R}_{n}$ and $\mathcal{B U}_{n}$ finitely based for $n \geq 3$ ?

The involutory semigroups $\mathcal{B R}_{2}$ and $\mathcal{B U}_{2}$ are easily seen to be finitely based. The finite basis problem for the plain semigroups $\left\langle B R_{n}, \cdot\right\rangle$ and $\left\langle B U_{n}, \cdot\right\rangle$ has been solved by the third author [55].

Acknowledgments. The second author was supported by Grant No. 174019 of the Ministry of Science and Technological Development of the Republic of Serbia. The third author acknowledges support from the Ministry for Education and Science of Russia, grant 2.1.1/13995, and from the Russian Foundation for Basic Research, grant 10-01-00524. 


\section{References}

[1] Amitsur, S. A.: Polynomial identities. Israel J. Math. 19, 183-199 (1974) Zbl 0297.16009 MR 0422335

[2] Araújo, J., Mitchell, J. D.: An elementary proof that every singular matrix is a product of idempotent matrices. Amer. Math. Monthly 112, 641-645 (2005) Zbl 1124.15006 MR 2158897

[3] Auinger, K.: Strict regular $*$-semigroups. In: Proceedings of the Conference on Semigroups with Applications, J. M. Howie et al. (eds.), World Sci., Singapore, 190-204 (1992) MR 1197855

[4] Ben-Israel, A., Greville, Th.: Generalized Inverses: Theory and Applications. Springer, Berlin (2003) Zbl 1026.15004 MR 1987382

[5] Burris, S., Sankappanavar, H. P.: A Course in Universal Algebra. Springer, Berlin (1981) Zbl 0478.08001 MR 0648287

[6] Clifford, A. H., Preston, G. B.: The Algebraic Theory of Semigroups, Vol. I. Amer. Math. Soc., Providence (1961) Zbl 0111.03403 MR 0132791

[7] Cline, R. E.: Note on the generalized inverse of the product of matrices. SIAM Rev. 6, 57-58 (1964) Zbl 0121.26105 MR 0161872

[8] Colombo, J., Koshlukov, P.: Central polynomials in the matrix algebra of order two. Linear Algebra Appl. 377, 53-67 (2004) Zbl 1044.16016 MR 2021602

[9] D'Amour, A., Racine, M.: *-Polynomial identites of matrices with the transpose involution: the low degrees. Trans. Amer. Math. Soc. 351, 5089-5106 (1999) Zbl 0935.16013 MR 1603886

[10] D'Amour, A., Racine, M.: *-Polynomial identites of matrices with the symplectic involution: the low degrees. Comm. Algebra 32, 895-918 (2004) Zbl 1068.16029 MR 2063788

[11] Dolinka, I.: On identities of finite involution semigroups. Semigroup Forum 80, 105-120 (2010) Zbl 1192.20042 MR 2580613

[12] Drazin, M. P.: Regular semigroups with involution. In: Proceedings of the Symposium on Regular Semigroups, Northern Illinois Univ., De Kalb, IL, 29-46 (1979) Zbl 0456.20032

[13] Drensky, V. S.: A minimal basis of identities for a second-order matrix algebra over a field of characteristic 0. Algebra i Logika 20, 282-290 (1981) (in Russian); English transl.: Algebra and Logic 20, 188-194 (1982) Zbl 0496.16017

[14] Drensky, V. S., Formanek, E.: Polynomial Identity Rings. Birkhäuser, Basel (2004) Zbl 1077.16025 MR 2064082

[15] Drensky, V. S., Giambruno, A.: On the *-polynomial identities of minimal degree for matrices with involution. Boll. Un. Mat. Ital. (A) 9, 471-482 (1995) Zbl 0862.16015 MR 1363547

[16] Erdos, J. A.: On products of idempotent matrices. Glasgow Math. J. 8, 118-122 (1967) Zbl 0157.07101 MR 0220751

[17] Genov, G. K.: A basis of identities of the algebra of third-order matrices over a finite field. Algebra i Logika 20, 385-388 (1981) (in Russian); English transl.: Algebra and Logic 20, 241-257 (1982) Zbl 0493.16012 MR 0663056

[18] Genov, G. K., Siderov, P. N.: A basis of the identities of the fourth order matrix algebra over a finite field. I, II. Serdica 8, 313-323, 351-366 (1982) (in Russian) Zbl 0515.16006 MR 0694958

[19] Gerhard, J. A., Petrich, M.: Free involutorial completely simple semigroups. Canad. J. Math. 37, 271-295 (1985) Zbl 0552.20040 MR 0780625

[20] Giambruno, A.: On *-polynomial identities for $n \times n$-matrices. J. Algebra 133, 433-438 (1990) Zbl 0725.16019 MR 1067416

[21] Giambruno, A., Zaicev, M.: Polynomial Identities and Asymptotic Methods. Amer. Math. Soc., Providence (2005) Zbl 1105.16001 MR 2176105 
[22] Golubchik, I. Z., Mikhalev, A. V.: A note on varieties of semiprime rings with semigroup identities. J. Algebra 54, 42-45 (1978) Zbl 0389.16006 MR 0511456

[23] Kanel-Belov, A., Rowen, L. H.: Computational Aspects of Polynomial Identities. A K Peters, Wellesley (2005) Zbl 1076.16018 MR 2124127

[24] Kemer, A. R.: The finite basis property of identities of associative algebras. Algebra i Logika 26, 597-641 (1987) (in Russian); English transl.: Algebra and Logic 26, 362-397 (1987) Zbl 0646.16013 MR 0985840

[25] Kemer, A. R.: Ideals of Identities of Associative Algebras. Amer. Math. Soc., Providence (1991) Zbl 0732.16001 MR 1108620

[26] Khalezov, E. A.: Isomorphisms of matrix semigroups. Ivanov. Gos. Ped. Inst. Uchenye Zap. Fiz.-Mat. Nauki 5, 42-56 (1954) (in Russian) Zbl 0059.01903 MR 0075957

[27] Kim, K. H., Roush, F.: On groups in varieties of semigroups. Semigroup Forum 16, 201-202 (1978) Zbl 0398.20060 MR 0480818

[28] Kleiman, E. I.: Bases of identities of varieties of inverse semigroups. Sibirsk Mat. Zh. 20, 760 777 (1979) (in Russian); English transl.: Sib. Math. J. 20, 530-543 (1979) Zbl 0417.20051 MR 0546267

[29] Koshlukov, P.: Basis of the identities of the matrix algebra of order two over a field of characteristic $p \neq 2$. J. Algebra 241, 410-434 (2001) Zbl 0988.16015 MR 1838859

[30] Kruse, R. L.: Identities satisfied by a finite ring. J. Algebra 26, 298-318 (1973) Zbl 0276.16014 MR 0325678

[31] Li, J. R., Luo, Y. F.: On the finite basis problem for the monoids of triangular boolean matrices. Algebra Universalis 65, 353-362 (2011) Zbl pre05939543 MR 2817557

[32] Lidl, R., Niederreiter, H.: Finite Fields. Cambridge Univ. Press, Cambridge (1997) Zbl 0866.11069 MR 1429394

[33] L'vov, I. V.: Varieties of associative rings. I. Algebra i Logika 12, 269-297 (1973) (in Russian); English transl.: Algebra and Logic 12, 150-167 (1973) Zbl 0288.16008 MR 0389973

[34] Magnus, W., Karras, A., Solitar, D.: Combinatorial Group Theory. Wiley, New York (1966) Zbl 0138.25604 MR 0207802

[35] Maltsev, Yu. N., Kuzmin, E. N.: A basis for the identities of the algebra of second-order matrices over a finite field. Algebra i Logika 17, 28-32 (1978) (in Russian); English transl.: Algebra and Logic 17, 18-21 (1978) Zbl 0395.16014 MR 0516388

[36] Margolis, S. W., Sapir, M. V.: Quasi-identities of finite semigroups and symbolic dynamics. Israel J. Math. 92, 317-331 (1995) Zbl 0840.2006 MR 1357761

[37] Meyer, C. D.: Matrix Analysis and Applied Linear Algebra. SIAM, Philadelphia (2000) Zbl 0962.15001 MR 1777382

[38] Moore, E. H.: On the reciprocal of the general algebraic matrix. Bull. Amer. Math. Soc. 26, 394-395 (1920)

[39] Neumann, H.: Varieties of Groups. Springer, Berlin (1967) Zbl 0251.20001 MR 0215899

[40] Oates, S., Powell, M. B.: Identical relations in finite groups. J. Algebra 1, 11-39 (1964) Zbl 0121.27202 MR 0161904

[41] Penrose, R.: A generalized inverse for matrices. Proc. Cambridge Philos. Soc. 51, 406-413 (1955) Zbl 0065.24603 MR 0069793

[42] Pin, J.-E., Straubing, H.: Monoids of upper triangular matrices. In: Semigroups. Structure and Universal Algebraic Problems, Gy. Pollák et al. (eds.), Colloq. Math. Soc. János Bolyai 39, North-Holland, Amsterdam, 259-272 (1985) Zbl 0635.20028 MR 0873155

[43] Procesi, C.: The invariant theory of $n \times n$ matrices. Adv. Math. 19, 306-381 (1976) Zbl 0331.15021 MR 0419491

[44] Procesi, C.: Lie Groups: an Approach through Invariants and Representations. Springer, Berlin (2007) Zbl 1154.22001 MR 2265844 
[45] Razmyslov, Yu. P.: Finite basing of the identities of a matrix algebra of second order over a field of characteristic zero. Algebra i Logika 12, 83-113 (1973) (in Russian); English transl.: Algebra and Logic 12, 47-63 (1973) Zbl 0282.17003 MR 0340348

[46] Rowen, L. H.: Polynomial Identities in Ring Theory. Academic Press, New York (1980) Zbl 0461.16001 MR 0576061

[47] Sapir, M. V.: Inherently non-finitely based finite semigroups. Mat. Sb. 133, 154-166 (1987) (in Russian); English transl.: Math. USSR-Sb. 61, 155-166 (1988) Zbl 0655.20045 MR 0905002

[48] Sapir, M. V.: Problems of Burnside type and the finite basis property in varieties of semigroups. Izv. Akad. Nauk SSSR, Ser. Mat. 51, 319-340 (1987) (in Russian); English transl.: Math. USSR-Izv. 30, 295-314 (1987) Zbl 0646.20047 MR 0897000

[49] Sapir, M. V.: Identities of finite inverse semigroups. Int. J. Algebra Comput. 3, 115-124 (1993) Zbl 0784.20032 MR 1214009

[50] Sapir, M. V.: Combinatorics on Words with Applications. IBP-Litp 1995/32: Rapport de Recherche Litp, Université Paris 7 (1995); http://www.math.vanderbilt.edu/ msapir/ftp/ course/course.pdf

[51] Schwarz, Š.: The semigroup of fully indecomposable relations and Hall relations. Czechoslovak Math. J. 23, 151-163 (1973) Zbl 0261.20057 MR 0316612

[52] Serre, J.-P.: Cours d'Arithmétique. Presses Universitaires de France, Paris (1970) Zbl 0225.12002 MR 0255476

[53] Volkov, M. V.: On finite basedness of semigroup varieties. Mat. Zametki 45, 12-23 (1989) (in Russian); English transl.: Math. Notes 45, 187-194 (1989) Zbl 0692.20048 MR 1001692

[54] Volkov, M. V.: The finite basis problem for finite semigroups. Sci. Math. Japan 53, 171-199 (2001) Zbl 0990.20039 MR 1821612

[55] Volkov, M. V.: Reflexive relations, extensive transformations and piecewise testable languages of a given height. Int. J. Algebra Comput. 14, 817-827 (2004) Zbl 1074.20036 MR 2104784

[56] Volkov, M. V., Goldberg, I. A.: The finite basis problem for monoids of triangular boolean matrices. In: Algebraic Systems, Formal Languages, and Conventional and Unconventional Computation Theory, Surikaisekikenkyusho Kokyuroku 1366, Res. Inst. Math. Sci., Kyoto Univ., Kyoto, 205-214 (2004) 